\title{
Finite difference approximations of multidimensional unsteady convection-diffusion-reaction equations
}

\author{
Adem Kaya \\ Department of Mathematics, Izmir Institute of Technology, 35430 Izmir, Turkey
}

\section{A R T I C L E I N F O}

\section{Article history:}

Received 3 March 2014

Received in revised form 19 December 2014

Accepted 15 January 2015

Available online 20 January 2015

\section{Keywords:}

Finite difference method

Finite element method

Unsteady convection-diffusion-reaction

\begin{abstract}
A B S T R A C T
In this paper, the numerical approximation of unsteady convection-diffusion-reaction equations with finite difference method on a special grid is studied in the convection or reaction-dominated regime. We extend the method [19] which was designed for multidimensional steady convection-diffusion-reaction equations to unsteady problems. We investigate two possible different ways of combining the discretization in time and in space (where the sequence of the discretizations is interchanged). Discretization in time is performed by using Crank-Nicolson and Backward-Euler finite difference schemes, while for the space discretization we consider the method [19]. Numerical tests are presented to show good performance of the method.
\end{abstract}

(c) 2015 Elsevier Inc. All rights reserved.

\section{Introduction}

Numerical solution of unsteady convection-diffusion-reaction (CDR) equations is an active research area. It is well known that when convection or reaction dominates standard Galerkin finite element method and central finite difference scheme produce undesired oscillations that pollute whole domain and stabilized methods have to be utilized. Within finite difference approaches, upwinding is one of the earliest and simple one to deal with convection-dominated problems. In the last few decades, essentially non-oscillatory (ENO) [17] and weighted ENO (WENO) [18] schemes have been developed in the context of hyperbolic partial differential equations. These schemes use a wider stencil than the simple upwinding. A comprehensive comparison of these methods was studied in [16]. Within finite element methods, one of the early attempts is streamlineupwind Petrov-Galerkin (SUPG) method which is first proposed in [6]. It is widely employed for many different applications, however, possible drawbacks of the method are its complexity and the presence of a stabilizing parameter that needs to be properly tuned for each problem. A more recent approach is the residual-free bubble method (RFB) which is based on enriching the finite element space. It is first studied in [2] to find a suitable value of stabilizing parameter for SUPG method. The main problem with this method is that it requires the solution of a local PDE. Inexpensive approximate solutions to this local problem were designed by several researchers [3-5,7,8]. Stabilized finite element methods for unsteady convectiondiffusion-reaction equations have been studied by several researchers. We refer to $[11,14,15]$ for different techniques. It is worth noting that recently adaptive methods were introduced for unsteady convection-diffusion-reaction equations [9,10].

In connection with the RFB method and based on the idea of the augmented space, Link-Cutting Bubble (LCB) strategy has been proposed (see [3]). It has been designed for one-dimensional convection-diffusion-reaction equations, especially to treat the most interesting cases of convection-dominated or reaction-dominated. It has been extended to one-dimensional

E-mail address: ademkaya@iyte.edu.tr. 
unsteady convection-diffusion-reaction equation in [1]. Robustness and accuracy of LCB strategy in all regimes allow to employ two types of partial differentiation of different nature; method of lines and horizontal method of lines (Rothe method).

We have proposed a new finite difference method for multidimensional steady convection-diffusion-reaction equations which adapts very well to all regimes in [19]. There, we did a simple splitting of convection-diffusion-reaction equations into one-dimensional convection-diffusion-reaction equations. The same splitting was done in [13]. We can consider the method [19] as an unusual extension of LCB strategy to higher dimensions. We refer to [19] for more information. In this paper, we will extend this approach to multidimensional unsteady convection-diffusion-reaction equations. We will apply both the method of lines and the horizontal method of lines.

The outline of the paper is as follows. In Section 2, stabilization technique for the steady problem [19] is given. In Section 3, we extend the method to unsteady convection-diffusion-reaction equations. Numerical tests are illustrated in Section 4 to show the performance of the method. Finally, concluding remarks.

\section{Stabilization technique for the steady problem [19]}

We start with considering the following one dimensional linear elliptic convection-diffusion-reaction problem on unit interval $I=(0,1)$

$$
\left\{\begin{array}{l}
\mathcal{L} u=-\epsilon u^{\prime \prime}+\beta u^{\prime}+\sigma u=f(x) \text { on } I, \\
u(0)=u(1)=0,
\end{array}\right.
$$

under the assumptions that the diffusion coefficient $\epsilon$ is positive constant, convection field $\beta$ and reaction field $\sigma$ are non-negative constants. $T_{h}=\left\{K_{k}\right\}$ denotes the decomposition of $I$ into subintervals and $h_{k}=x_{k}-x_{k-1}$ size of the interval $K_{k}$, where $K_{k}=\left(x_{k-1}, x_{k}\right), k=1, \ldots, N$. Finite difference correspondence of Galerkin finite element method with piecewise linear basis functions on non-uniform mesh for problem (1) is as follows:

$$
\left\{\begin{array}{l}
-\epsilon \frac{u_{j+1}}{h_{2}}+\epsilon \frac{u_{j}}{h_{2}}+\epsilon \frac{u_{j}}{h_{1}}-\epsilon \frac{u_{j-1}}{h_{1}}+\beta \frac{u_{j+1}-u_{j-1}}{2}+\sigma \frac{h_{2} u_{j+1}+2 h_{2} u_{j}+2 h_{1} u_{j}+h_{1} u_{j-1}}{6} \\
=\frac{h_{2} f_{j+1}+2 h_{2} f_{j}+2 h_{1} f_{j}+h_{1} f_{j-1}}{6}, \\
u_{0}=u_{N}=0, \quad j=2, \ldots, N-1,
\end{array}\right.
$$

where $h_{1}=x_{j}-x_{j-1}$ and $h_{2}=x_{j+1}-x_{j}$. Our aim is to mimic the stabilizing effect of LCB strategy [3]. To do this, we choose two sub-grid nodes say $z_{1}$ and $z_{2}$ in a typical element $\left(x_{1}, x_{2}\right)$ which are defined as follows:

$$
\begin{aligned}
& z_{1}-x_{1}=\min \left\{h-2\left(x_{2}-z_{2}\right), \frac{3 \beta+\sqrt{9 \beta^{2}+24 \epsilon \sigma}}{2 \sigma}\right\}, \\
& x_{2}-z_{2}=\min \left\{\frac{h}{3}, \frac{-3 \beta+\sqrt{9 \beta^{2}+24 \epsilon \sigma}}{2 \sigma}\right\}
\end{aligned}
$$

where $h=x_{2}-x_{1}$. Once the sub-grid nodes are constructed, the finite difference scheme (2) gives the same result with LCB strategy on modified discretization. In two space dimensions, we consider the following constant coefficient linear elliptic convection-diffusion-reaction problem in a polygonal domain $\Omega$;

$$
\left\{\begin{array}{l}
-\epsilon\left(\frac{\partial^{2} u}{\partial x^{2}}+\frac{\partial^{2} u}{\partial y^{2}}\right)+b \cdot\left(\frac{\partial u}{\partial x}, \frac{\partial u}{\partial y}\right)+\sigma u=f(x, y) \text { on } \Omega, \\
u(x, y)=g \text { on } \partial \Omega
\end{array}\right.
$$

under the assumptions that $\epsilon>0, b=\left(b_{1}, b_{2}\right)^{T} \neq 0$ and $\sigma \geq 0$. Eq. (4) can be rewritten as follows:

$$
\left\{\begin{array}{l}
-\epsilon\left(\frac{\partial^{2} u}{\partial x^{2}}\right)+b_{1}\left(\frac{\partial u}{\partial x}\right)+\sigma \frac{\left|b_{1}\right|}{\left|b_{1}\right|+\left|b_{2}\right|} u-\epsilon\left(\frac{\partial^{2} u}{\partial y^{2}}\right)+b_{2}\left(\frac{\partial u}{\partial y}\right)+\sigma \frac{\left|b_{2}\right|}{\left|b_{1}\right|+\left|b_{2}\right|} u \\
=\frac{\left|b_{1}\right|}{\left|b_{1}\right|+\left|b_{2}\right|} f(x, y)+\frac{\left|b_{2}\right|}{\left|b_{1}\right|+\left|b_{2}\right|} f(x, y) \text { on } \Omega, \\
u(x, y)=g \text { on } \partial \Omega .
\end{array}\right.
$$

Next, we split Eq. (5) into two separate one dimensional convection-diffusion-reaction equations as in [13],

$$
\left\{\begin{array}{l}
-\epsilon\left(\frac{d^{2} u}{d x^{2}}\right)+b_{1}\left(\frac{d u}{d x}\right)+\sigma \frac{\left|b_{1}\right|}{\left|b_{1}\right|+\left|b_{2}\right|} u=\frac{\left|b_{1}\right|}{\left|b_{1}\right|+\left|b_{2}\right|} f(x, y), \\
-\epsilon\left(\frac{d^{2} u}{d y^{2}}\right)+b_{2}\left(\frac{d u}{d y}\right)+\sigma \frac{\left|b_{2}\right|}{\left|b_{1}\right|+\left|b_{2}\right|} u=\frac{\left|b_{2}\right|}{\left|b_{1}\right|+\left|b_{2}\right|} f(x, y) .
\end{array}\right.
$$


Now, we apply the finite difference scheme (2) to the equations in (6) and sum up these discretizations to get the following finite difference approximation for Eq. (4);

$$
\begin{aligned}
-\epsilon & \frac{u_{i+1, j}}{n_{2}}+\epsilon \frac{u_{i, j}}{n_{2}}+\epsilon \frac{u_{i, j}}{n_{1}}-\epsilon \frac{u_{i-1, j}}{n_{1}}+b_{1} \frac{u_{i+1, j}-u_{i-1, j}}{2} \\
& +\sigma \frac{\left|b_{1}\right|}{\left|b_{1}\right|+\left|b_{2}\right|} \frac{n_{2} u_{i+1, j}+2 n_{2} u_{i, j}+2 n_{1} u_{i, j}+n_{1} u_{i-1, j}}{6}-\epsilon \frac{u_{i, j+1}}{m_{2}}+\epsilon \frac{u_{i, j}}{m_{2}}+\epsilon \frac{u_{i, j}}{m_{1}}-\epsilon \frac{u_{i, j-1}}{m_{1}} \\
& +b_{2} \frac{u_{i, j+1}-u_{i, j-1}}{2}+\sigma \frac{\left|b_{2}\right|}{\left|b_{1}\right|+\left|b_{2}\right|} \frac{m_{2} u_{i, j+1}+2 m_{2} u_{i, j}+2 m_{1} u_{i, j}+m_{1} u_{i, j-1}}{6} \\
= & \frac{\left|b_{1}\right|}{\left|b_{1}\right|+\left|b_{2}\right|} \frac{n_{2} f_{i+1, j}+2 n_{2} f_{i, j}+2 n_{1} f_{i, j}+n_{1} f_{i-1, j}}{6} \\
& +\frac{\left|b_{2}\right|}{\left|b_{1}\right|+\left|b_{2}\right|} \frac{m_{2} f_{i, j+1}+2 m_{2} f_{i, j}+2 m_{1} f_{i, j}+m_{1} f_{i, j-1}}{6}
\end{aligned}
$$

where $u_{i, j} \approx u\left(x_{i}, y_{j}\right), n_{2}=x_{i+1}-x_{i}, n_{1}=x_{i}-x_{i-1}, m_{2}=y_{i+1}-y_{i}, m_{1}=y_{i}-y_{i-1}, i=2, N-1$ and $j=2, M-1$.

As for the three dimensional convection-diffusion-reaction problem, we consider the following equation;

$$
\left\{\begin{array}{l}
-\epsilon\left(\frac{\partial^{2} u}{\partial x^{2}}+\frac{\partial^{2} u}{\partial y^{2}}+\frac{\partial^{2} u}{\partial z^{2}}\right)+b \cdot\left(\frac{\partial u}{\partial x}, \frac{\partial u}{\partial y}, \frac{\partial u}{\partial z}\right)+\sigma u=f(x, y, z) \quad \text { on } \Omega, \\
u(x, y, z)=g \text { on } \partial \Omega
\end{array}\right.
$$

where $\epsilon>0, b=\left(b_{1}, b_{2}, b_{3}\right)^{T} \neq 0$ and $\sigma \geq 0$. Doing the same arrangement we did for Eq. (4), to Eq. (8) we get

$$
\left\{\begin{array}{l}
-\epsilon\left(\frac{\partial^{2} u}{\partial x^{2}}\right)+b_{1}\left(\frac{\partial u}{\partial x}\right)+\sigma \frac{\left|b_{1}\right|}{\left|b_{1}\right|+\left|b_{2}\right|+\left|b_{3}\right|} u-\epsilon\left(\frac{\partial^{2} u}{\partial y^{2}}\right)+b_{2}\left(\frac{\partial u}{\partial y}\right) \\
+\sigma \frac{\left|b_{2}\right|}{\left|b_{1}\right|+\left|b_{2}\right|+\left|b_{3}\right|} u-\epsilon\left(\frac{\partial^{2} u}{\partial z^{2}}\right)+b_{3}\left(\frac{\partial u}{\partial z}\right)+\sigma \frac{\left|b_{3}\right|}{\left|b_{1}\right|+\left|b_{2}\right|+\left|b_{3}\right|} u \\
=\frac{\left|b_{1}\right|}{\left|b_{1}\right|+\left|b_{2}\right|+\left|b_{3}\right|} f(x, y, z)+\frac{\left|b_{2}\right|}{\left|b_{1}\right|+\left|b_{2}\right|+\left|b_{3}\right|} f(x, y, z)+\frac{\left|b_{3}\right|}{\left|b_{1}\right|+\left|b_{2}\right|+\left|b_{3}\right|} f(x, y, z) \text { on } \Omega, \\
u(x, y, z)=g \text { on } \partial \Omega
\end{array}\right.
$$

which can be considered as a sum of separate three one dimensional convection-diffusion-reaction equations,

$$
\begin{aligned}
& -\epsilon\left(\frac{d^{2} u}{d x^{2}}\right)+b_{1}\left(\frac{d u}{d x}\right)+\sigma \frac{\left|b_{1}\right|}{\left|b_{1}\right|+\left|b_{2}\right|+\left|b_{3}\right|} u=\frac{\left|b_{1}\right|}{\left|b_{1}\right|+\left|b_{2}\right|+\left|b_{3}\right|} f(x, y, z), \\
& -\epsilon\left(\frac{d^{2} u}{d y^{2}}\right)+b_{2}\left(\frac{d u}{d y}\right)+\sigma \frac{\left|b_{2}\right|}{\left|b_{1}\right|+\left|b_{2}\right|+\left|b_{3}\right|} u=\frac{\left|b_{2}\right|}{\left|b_{1}\right|+\left|b_{2}\right|+\left|b_{3}\right|} f(x, y, z), \\
& -\epsilon\left(\frac{d^{2} u}{d z^{2}}\right)+b_{3}\left(\frac{d u}{d z}\right)+\sigma \frac{\left|b_{3}\right|}{\left|b_{1}\right|+\left|b_{2}\right|+\left|b_{3}\right|} u=\frac{\left|b_{3}\right|}{\left|b_{1}\right|+\left|b_{2}\right|+\left|b_{3}\right|} f(x, y, z) .
\end{aligned}
$$

Applying the finite difference scheme (2) to the equations in (10) and summing up these three discretizations we get the following approximate form for the problem (8);

$$
\begin{aligned}
-\epsilon & \frac{u_{i+1, j, k}}{n_{2}}+\epsilon \frac{u_{i, j, k}}{n_{2}}+\epsilon \frac{u_{i, j, k}}{n_{1}}-\epsilon \frac{u_{i-1, j, k}}{n_{1}}+b_{1} \frac{u_{i+1, j, k}-u_{i-1, j, k}}{2} \\
& +\sigma \frac{\left|b_{1}\right|}{\left|b_{1}\right|+\left|b_{2}\right|+\left|b_{3}\right|} \frac{n_{2} u_{i+1, j, k}+2 n_{2} u_{i, j, k}+2 n_{1} u_{i, j, k}+n_{1} u_{i-1, j, k}}{6} \\
& -\epsilon \frac{u_{i, j+1, k}}{m_{2}}+\epsilon \frac{u_{i, j, k}}{m_{2}}+\epsilon \frac{u_{i, j, k}}{m_{1}}-\epsilon \frac{u_{i, j-1, k}}{m_{1}}+b_{2} \frac{u_{i, j+1, k}-u_{i, j-1, k}}{2} \\
& +\sigma \frac{\left|b_{2}\right|}{\left|b_{1}\right|+\left|b_{2}\right|+\left|b_{3}\right|} \frac{m_{2} u_{i, j+1, k}+2 m_{2} u_{i, j, k}+2 m_{1} u_{i, j, k}+m_{1} u_{i, j-1, k}}{6} \\
& -\epsilon \frac{u_{i, j, k+1}}{k_{2}}+\epsilon \frac{u_{i, j, k}}{k_{2}}+\epsilon \frac{u_{i, j, k}}{k_{1}}-\epsilon \frac{u_{i, j, k-1}}{k_{1}}+b_{3} \frac{u_{i, j, k+1}-u_{i, j, k-1}}{2} \\
& +\sigma \frac{\left|b_{3}\right|}{\left|b_{1}\right|+\left|b_{2}\right|+\left|b_{3}\right|} \frac{k_{2} u_{i, j, k+1}+2 k_{2} u_{i, j, k}+2 k_{1} u_{i, j, k}+k_{1} u_{i, j, k-1}}{6}
\end{aligned}
$$



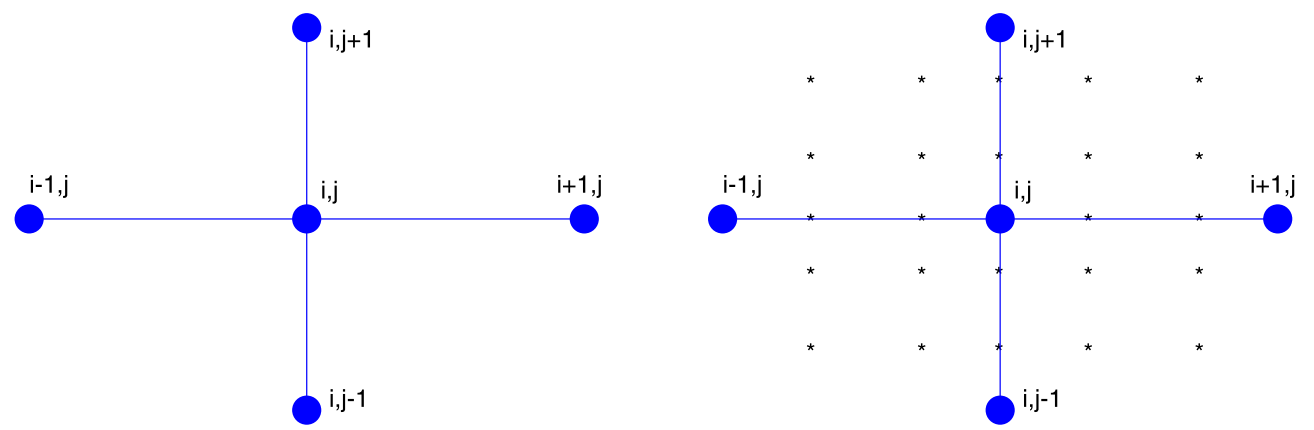

Fig. 1. Five point stencil (left) and its modified version (right) in $2 D$.

$$
\begin{aligned}
= & \frac{\left|b_{1}\right|}{\left|b_{1}\right|+\left|b_{2}\right|+\left|b_{3}\right|} \frac{n_{2} f_{i+1, j, k}+2 n_{2} f_{i, j, k}+2 n_{1} f_{i, j, k}+n_{1} f_{i-1, j, k}}{6} \\
& +\frac{\left|b_{2}\right|}{\left|b_{1}\right|+\left|b_{2}\right|+\left|b_{3}\right|} \frac{m_{2} f_{i, j+1, k}+2 m_{2} f_{i, j, k}+2 m_{1} f_{i, j, k}+m_{1} f_{i, j-1, k}}{6} \\
& +\frac{\left|b_{3}\right|}{\left|b_{1}\right|+\left|b_{2}\right|+\left|b_{3}\right|} \frac{k_{2} f_{i, j, k+1}+2 k_{2} f_{i, j, k}+2 k_{1} f_{i, j, k}+k_{1} f_{i, j, k-1}}{6}
\end{aligned}
$$

where $i=2, \ldots, N-1, j=2, \ldots, M-1, k=2, \ldots, K-1, n_{2}=x_{i+1}-x_{i}, n_{1}=x_{i}-x_{i-1}, m_{2}=y_{i+1}-y_{i}, m_{1}=y_{i}-y_{i-1}$, $k_{2}=z_{i+1}-z_{i}, k_{1}=z_{i}-z_{i-1}$ and $u_{i, j, k} \approx u\left(x_{i}, y_{j}, z_{k}\right)$.

\subsection{Derivation of the finite difference schemes on non-uniform mesh with Taylor series expansion}

We have provided a simple way to approximate the derivatives and function from Taylor series expansion on non-uniform mesh in [19]. Omitting the details, approximations are as follow;

$$
\begin{aligned}
& u^{\prime}(x) \approx \frac{u_{j+1}-u_{j-1}}{h_{1}+h_{2}}, \\
& u^{\prime \prime}(x) \approx\left(\frac{u_{j+1}}{h_{2}}-\frac{u_{j}}{h_{2}}-\frac{u_{j}}{h_{1}}+\frac{u_{j-1}}{h_{1}}\right)\left(\frac{2}{h_{1}+h_{2}}\right), \\
& u(x) \approx \frac{h_{2} u_{j+1}+h_{1} u_{j-1}}{h_{1}+h_{2}}, \\
& u(x) \approx \frac{h_{2} u_{j+1}+2 h_{2} u_{j}+2 h_{1} u_{j}+h_{1} u_{j-1}}{3\left(h_{1}+h_{2}\right)} .
\end{aligned}
$$

Approximating $u^{\prime \prime}$ by (12), $u^{\prime}$ by (11), $u$ and source function $f$ by (14) for the one dimensional convection-diffusion-reaction equation (1) and multiplying both sides of the approximation with $\left(h_{1}+h_{2}\right) / 2$ we get the finite difference approximation (2) which is second order accurate, in the sense $O\left(h_{1}^{2}\right)+O\left(h_{2}^{2}\right)$. At this point, we have two alternatives to approximate the multidimensional convection-diffusion-reaction equations. For the first case, we approximate the terms in the CDR equations by (11), (12) and (14) for which we obtain a first order accurate finite difference scheme. For the second case, we approximate with splitting the multidimensional CDR equation into one dimensional CDR equations, then we approximate the one dimensional CDR equations by (4), separately and sum up the approximations as I did. With the second approach, we get a second order accurate scheme, in the sense $O\left(n_{1}^{2}\right)+O\left(n_{2}^{2}\right)+O\left(m_{1}^{2}\right)+O\left(m_{2}^{2}\right)$ where $n_{2}=x_{i+1}-x_{i}, n_{1}=x_{i}-x_{i-1}$, $m_{2}=y_{i+1}-y_{i}, m_{1}=y_{i}-y_{i-1}$ for two space dimensional CDR equation. Under the assumption $n_{1}+n_{2}=m_{1}+m_{2}$ on non-uniform grid, one can get the second approximation from the first approach.

\subsection{Discretization of the domain}

Discretization of the domain consists of two steps. First we do an initial discretization that can be structured or unstructured. A five point stencil of the initial discretization is presented in Fig. 1 (left) in two space dimensions. Second (augmented) stencil is obtained by adding 24 sub-grid nodes into the initial stencil as shown in Fig. 1 (right). The $x-$ coordinates of the sub-grid nodes are calculated using (3) with the problem parameters of the first equation in (6) either in the interval $\left(x_{i-1, j}, x_{i, j}\right)$ or in $\left(x_{i, j}, x_{i+1, j}\right)$ according to their projection onto the $x$-axis. Similarly, $y$-coordinates are determined by applying the same procedure to the second equation in (6) either in the interval $\left(y_{i, j-1}, y_{i, j}\right)$ or in $\left(y_{i, j}, y_{i, j+1}\right)$ according to their projection onto the $y$-axis. In three space dimensions, 124 sub-grid nodes are added to get augmented stencil and locations of the sub-grid nodes are determined by applying the same procedure using the problem parameters 
of equations in (10). Note that since everything is done on stencil level, the method can be applied on complex domains. We refer to [19] to see example in complex domain.

In particular, we denote the number of grid points of initial discretization in $x, y$ and $z$-directions in a rectangular domain by $N, M$ and $K$, respectively. Points in the initial discretization are denoted by $\left(x_{i, j}, y_{i, j}\right)$ in $2 D$ and by $\left(x_{i, j, k}, y_{i, j, k}, z_{i, j, k}\right)$ in $3 D$ where $i=1, \ldots, N, j=1, \ldots, M, k=1, \ldots, K$. We note that the modified discretization consists of $(3 N-2) \times(3 M-2)$ points in $2 D$ and $(3 N-2) \times(3 M-2) \times(3 K-2)$ in $3 D$. Algorithm 1 summarizes the procedure in $2 D$ and Algorithm 2 in $3 D$.

Data: $\left(x_{i, j}, y_{i, j}\right)$ for $i=1, \ldots, N, j=1, \ldots, M$ and the problem parameters.

Result: $\left(x_{i, j}^{\prime}, y_{i, j}^{\prime}\right)$ where $i=1, \ldots, 3 N-2$ and $j=1, \ldots, 3 M-2$.

Step 1: Do an initial discretization that can be structured or unstructured.

Step 2: The derivation of $x_{i, j}^{\prime}$,

Use the problem parameters of the first equation in (6): $\epsilon=\epsilon, \beta=\left|b_{1}\right|$ and $\sigma=\sigma \frac{\left|b_{1}\right|}{\left|b_{1}\right|+\left|b_{2}\right|}$;

for $j=1$ to $3 M-2$ do ;

for $i=1$ to $N-1$ do ;

Using Eq. (3), calculate $z_{1}$ and $z_{2}$ in $\left(x_{i+1, j}, x_{i, j}\right)$ to get

$$
x_{3 i, j}^{\prime}=z_{2} \quad x_{3 i-1, j}^{\prime}=z_{1} \quad x_{3 i-2, j}^{\prime}=x_{i, j} \quad x_{3 N-2, j}^{\prime}=x_{N, j} .
$$

Step 3: The derivation of $y_{i, j}^{\prime}$;

Use the problem parameters of the second equation in (6): $\epsilon=\epsilon, \beta=\left|b_{2}\right|$ and $\sigma=\sigma \frac{\left|b_{2}\right|}{\left|b_{1}\right|+\left|b_{2}\right|}$;

for $j=1$ to $3 N-2$ do ;

for $i=1$ to $M-1$ do ;

Using Eq. (3), calculate $z_{1}$ and $z_{2}$ in $\left(y_{j, i+1}, y_{j, i}\right)$ to get

$$
y_{j, 3 i}^{\prime}=z_{2} \quad y_{j, 3 i-1}^{\prime}=z_{1} \quad y_{j, 3 i-2}^{\prime}=y_{j, i} \quad y_{j, 3 M-2}^{\prime}=y_{j, M}
$$

Algorithm 1: Discretization of the domain in $2 D$ in a rectangular domain.

Fig. 2 illustrates an initial discretization and augmented discretizations in $2 D$ obtained with Algorithm 1 . We first set $\epsilon=1,\left(b_{1}, b_{2}\right)=\left(\cos 45^{\circ}, \sin 45^{\circ}\right), \sigma=10^{-6}$ (which corresponds to diffusion-dominated case), then we set $\epsilon=0.01$, $\left(b_{1}, b_{2}\right)=\left(\cos 45^{\circ}, \sin 45^{\circ}\right), \sigma=10^{-6}$ (which corresponds to convection-dominated case), finally we set $\epsilon=0.01,\left(b_{1}, b_{2}\right)=$ $\left(\cos 45^{\circ}, \sin 45^{\circ}\right), \sigma=10$ (which corresponds to reaction-dominated case) to show samples of discretizations in three different regimes.

Note that the problem is first solved on augmented grid and then the sub-grid nodes are excluded to simulate the numerical results.

\section{The unsteady problem}

LCB strategy has been extended to unsteady convection-diffusion-reaction equations in one dimension in [1]. Two different types of partial differentiation of different nature; the method of lines and the horizontal method of lines were employed. The method of lines was referred as FEs_FDt and the horizontal method of lines was referred as FDt_FEs. Unlike [1], we will do finite difference discretization in space and we will refer to the method of lines as FDs_FDt and to the horizontal method of lines as FDt_FDs. We focus on the Crank-Nicolson and the Backward-Euler (one step BDF formula) finite difference schemes for the time integration. We will refer to the Crank-Nicolson scheme as $C N$ and to the Backward-Euler scheme as $B E$. For example, $C N_{-} F D s_{-} F D t$ means that the method of line is used with the Crank-Nicolson time discretization.

Let $\Omega \subset R^{d}, d \in\{2,3\}$ be a polygonal bounded domain and let $[0, T]$ be a finite time interval. The unsteady convectiondiffusion-reaction equation which will be considered in this paper is as follows:

$$
\begin{aligned}
& \partial_{t} u-\epsilon \Delta u+b . \nabla u+\sigma u=f \quad \text { in }(0, T] \times \Omega, \\
& u=g \quad \text { on } \partial \Omega, \\
& u(0, X)=u_{0}(X) \text { in } \Omega
\end{aligned}
$$

where $\epsilon>0, \sigma \geq 0$ and $b \neq 0$.

\subsection{First approach: FDt_FDs}

Consider a uniform partition $\left\{0=t_{0}<t_{1}<\ldots<t_{N}=T\right\}$ of the time interval $[0, T]$ with time-step size $\Delta t$. Then, time discretization of problem (15) by the Crank-Nicolson scheme gives 
Data: $\left(x_{i, j, k}, y_{i, j, k}, z_{i, j, k}\right)$ for $i=1, \ldots, N, j=1, \ldots, M, k=1, \ldots, K$ and the problem parameters.

Result: $\quad\left(x_{i, j, k}^{\prime}, y_{i, j, k}^{\prime}, z_{i, j, k}^{\prime}\right)$ for $i=1, \ldots, 3 N-2, j=1, \ldots, 3 M-2$ and $k=1, \ldots, 3 K-2$.

Step 1: Do an initial discretization that can be structured or unstructured.

Step 2: The derivation of $x_{i, j, k}^{\prime}$,

Use the problem parameters of the first equation in (10): $\epsilon=\epsilon, \beta=\left|b_{1}\right|, \sigma=\sigma \frac{\left|b_{1}\right|}{\left|b_{1}\right|+\left|b_{2}\right|+\left|b_{3}\right|}$ and use Eq. (3) to calculate $z_{1}, z_{2}$ in $\left(x_{i+1, j, k}, x_{i, j, k}\right)$ then,

for $k=1$ to $3 K-2$ do ;

for $j=1$ to $3 M-2$ do ;

for $i=1$ to $N-1$ do ;

$$
x_{3 i, j, k}^{\prime}=z_{2} \quad x_{3 i-1, j, k}^{\prime}=z_{1} \quad x_{3 i-2, j, k}^{\prime}=x_{i, j} \quad x_{3 N-2, j, k}^{\prime}=x_{N, j, k} .
$$

Step 3: The derivation of $y_{i, j, k}^{\prime}$,

Use the problem parameters of the second equation in (10): $\epsilon=\epsilon, \beta=\left|b_{2}\right|, \sigma=\sigma \frac{\left|b_{2}\right|}{\left|b_{1}\right|+\left|b_{2}\right|+\left|b_{3}\right|}$ and use Eq. (3) to calculate $z_{1}, z_{2}$ in $\left(y_{j, i+1, k}, y_{j, i, k}\right)$ then,

for $k=1$ to $3 K-2$ do ;

for $j=1$ to $3 N-2$ do ;

for $i=1$ to $M-1$ do ;

$$
y_{j, 3 i, k}^{\prime}=z_{2} \quad y_{j, 3 i-1, k}^{\prime}=z_{1} \quad y_{j, 3 i-2, k}^{\prime}=y_{j, i, k} \quad y_{j, 3 M-2, k}^{\prime}=y_{j, M, k} .
$$

Step 4 : The derivation of $z_{j, i, k}^{\prime}$,

Use the problem parameters of the third equation in (10): $\epsilon=\epsilon, \beta=\left|b_{3}\right|, \sigma=\sigma \frac{\left|b_{3}\right|}{\left|b_{1}\right|+\left|b_{2}\right|+\left|b_{3}\right|}$ and use Eq. (3) to calculate $z_{1}, z_{2}$ in $\left(z_{j, i, k+1}, z_{j, i, k}\right)$ then,

for $j=1$ to $3 N-2$ do ;

for $i=1$ to $3 M-2$ do ;

for $k=1$ to $K-1$ do ;

$$
z_{j, i, 3 k}^{\prime}=z_{2} \quad z_{j, i, 3 k-1}^{\prime}=z_{1} \quad z_{j, i, 3 k-2}^{\prime}=y_{j, i} \quad z_{j, i, 3 K-2}^{\prime}=y_{j, i, K} .
$$

Algorithm 2: Discretization of the domain in $3 D$ in a rectangular domain.
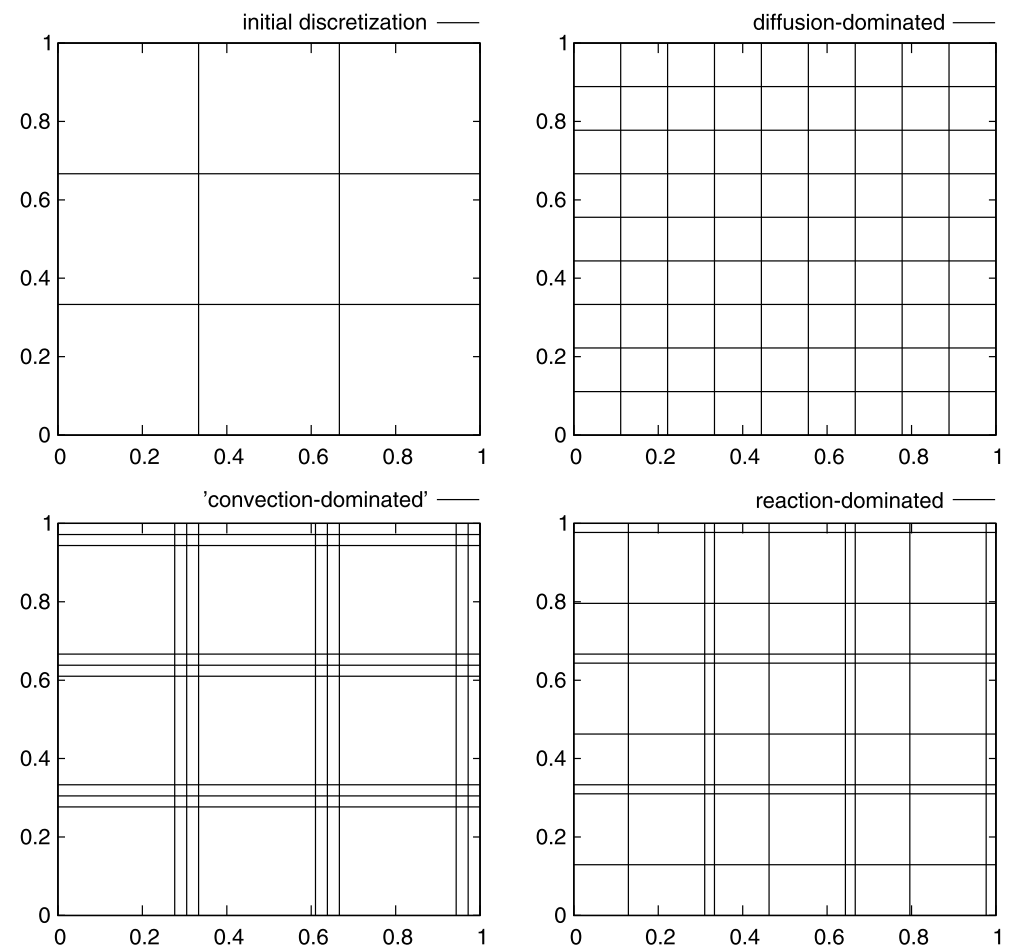

Fig. 2. Initial discretization and modified discretizations in three different regimes for $N=M=4$. 


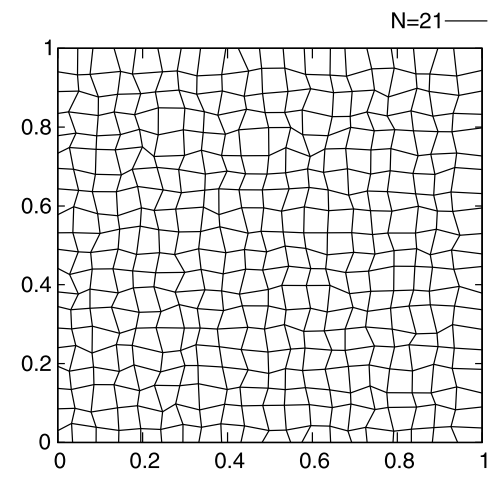

Fig. 3. Initial discretization of an unstructured grid.

$$
\begin{aligned}
& -\frac{\epsilon \Delta t}{2} \Delta u^{n+1}+\frac{\Delta t}{2} b \cdot \nabla u^{n+1}+\left(\frac{\sigma \Delta t}{2}+1\right) u^{n+1}=\frac{\epsilon \Delta t}{2} \Delta u^{n}-\frac{\Delta t}{2} b \cdot \nabla u^{n}-\left(\frac{\sigma \Delta t}{2}-1\right) u^{n}+\frac{\Delta t}{2}\left(f^{n+1}+f^{n}\right), \\
& n=0,1, \ldots, N-1, u^{0}=u_{0}
\end{aligned}
$$

which might be regarded as a family of steady advection-diffusion-reaction problems. Since $\epsilon \Delta t / 2, \frac{\Delta t}{2} b$ and $(\sigma \Delta t / 2+1)$ play the role of diffusion, advection and reaction coefficients, respectively, discretization of the domain is done by means of the algorithms given in Section 2 using these modified parameters. Time discretization of Eq. (15) with the Backward-Euler scheme gives

$$
\begin{aligned}
& -\epsilon \Delta t \Delta u^{n+1}+\Delta t b . \nabla u^{n+1}+(\Delta t \sigma+1) u^{n+1}=u^{n}+\Delta t f^{n}, \\
& n=0,1, \ldots, N-1, u^{0}=u_{0} .
\end{aligned}
$$

In this approach, $\epsilon \Delta t, b \Delta t$ and $(\sigma \Delta t+1)$ play the role of diffusion, advection and reaction coefficients, respectively. Hence, these modified problem parameters are used to calculate the sub-grid nodes. Once the time discretization is carried out, for each $n=0,1,2, \ldots, N-1$ we discretize problems (16) and (17) in space by means of the finite difference schemes reviewed in Section 2.

\subsection{Second approach: FDs_FDt}

In this approach, we first discretize in space by means of the finite difference scheme reviewed in Section 2. Discretization of the domain is done with the original problem parameters as in Section 2. Once the space discretization is carried out, we use the desired time discretization to get full discretized version of problem (15).

Observe that the final results of $F D t_{-} F D s$ and $F D s_{-} F D t$ discretizations are algebraically equivalent. The difference occurs in problem parameters that are used to calculate the sub-grid nodes.

\section{Numerical tests}

In this section, we report some numerical experiments to illustrate the performance of the present algorithm. $N$ represents the number of grid points in $x, y$ and $z$-directions throughout this section. We apply all tests in unit square in $2 D$ and in unit cube in $3 D$. The domains are discretized by considering both structured and unstructured grids. The unstructured grids are obtained by randomly perturbing the interior nodes of structured grids of initial discretization with coordinates $\left(x_{i}, y_{i}\right)$ as follows:

$$
x_{i}^{\prime \prime}=x_{i}-h_{1} \operatorname{rand}() / 2, \quad y_{i}^{\prime \prime}=y_{i}-h_{2} \operatorname{rand}() / 2
$$

where $\left(x_{i}^{\prime \prime}, y_{i}^{\prime \prime}\right)$ represents the corresponding coordinates of the unstructured grid, $h_{1}=x_{i+1}-x_{i}, h_{2}=y_{i+1}-y_{i}$ and rand() is a function that returns random numbers uniformly distributed in the interval $[0,1]$. Fig. 3 illustrates an example obtained with this procedure. In order to take cut planes parallel to $x y$-plane in $3 D$, I did not apply the perturbation in $z$-direction. MGMRES iterative solver was used to solve the linear system of equations and I did not experience any problems.

\subsection{Test 1}

The first example is the advection-diffusion-reaction equation where the reaction field is negligible $\left(\sigma=10^{-6}\right)$. The diffusion coefficient is set to $\epsilon=10^{-4}$, the convective field to $b=(1,0)$. There are no existing external forces acting on the system. We take a smooth initial condition which is defined as follows:

$$
u(0, x, y)=-10 e^{-\left(x^{2}+y^{2}\right) \log 2 / 0.09} \sin (3 x) \sin (\pi x) \cos \left(\frac{\pi}{2}+y\right) \cos \left(\frac{\pi y}{2}\right) .
$$



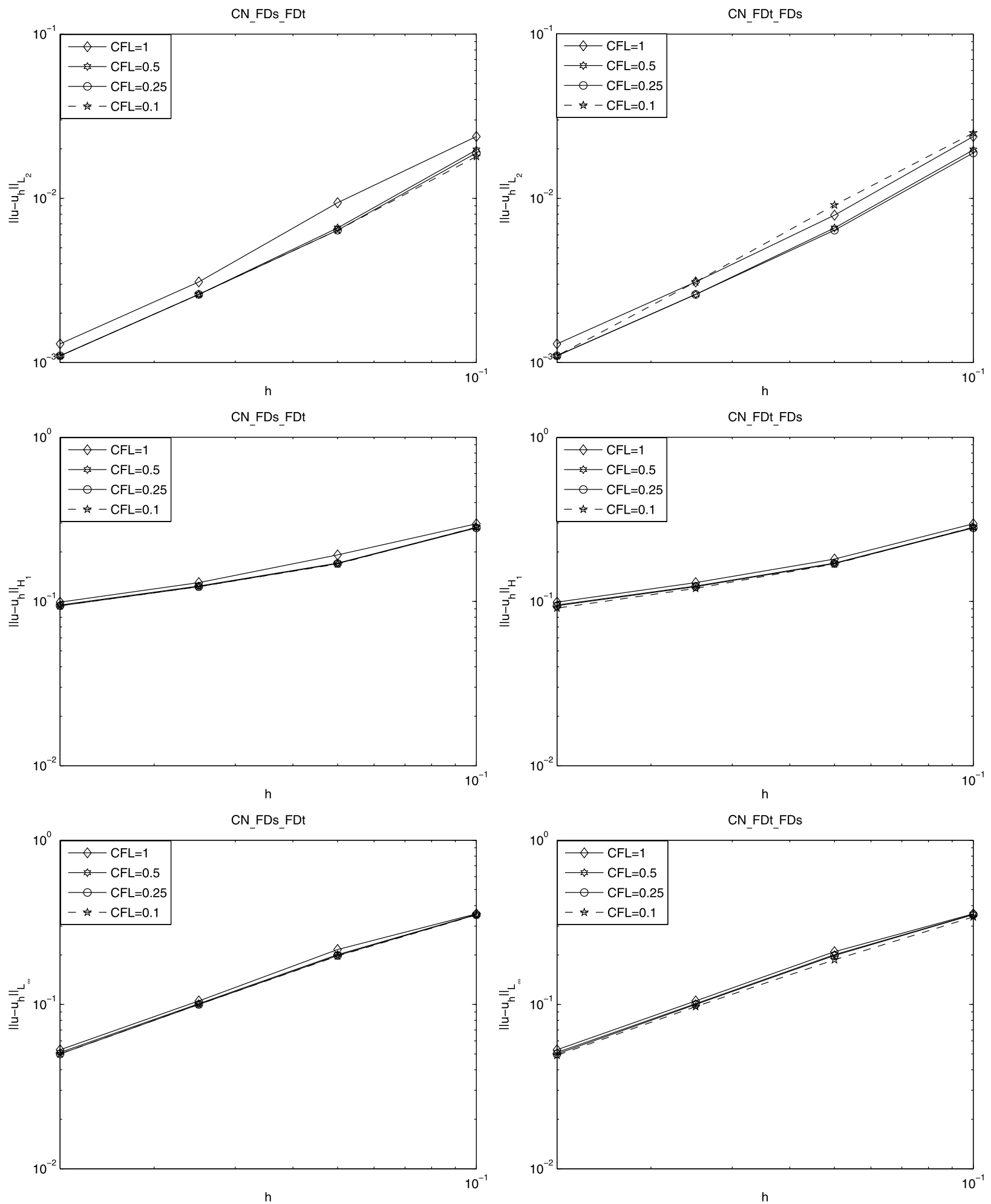

Fig. 4. Test 1 , convergence rates in $L_{2}, H_{1}$ and $L_{\infty}$ norms obtained with Crank-Nicolson time discretization.

The Crank-Nicolson and the Backward Euler time discretizations combined with FDs_FDt and FDt_FDs were employed. The exact solution of this problem was obtained with standard Galerkin method with 320000 triangular elements and with time step $\Delta t=0.0001$. Taking $h=0.1,0.05,0.025,0.0125$ uniform grid sizes in $x$ and $y$ directions, we found errors. Figs. 4, 5 

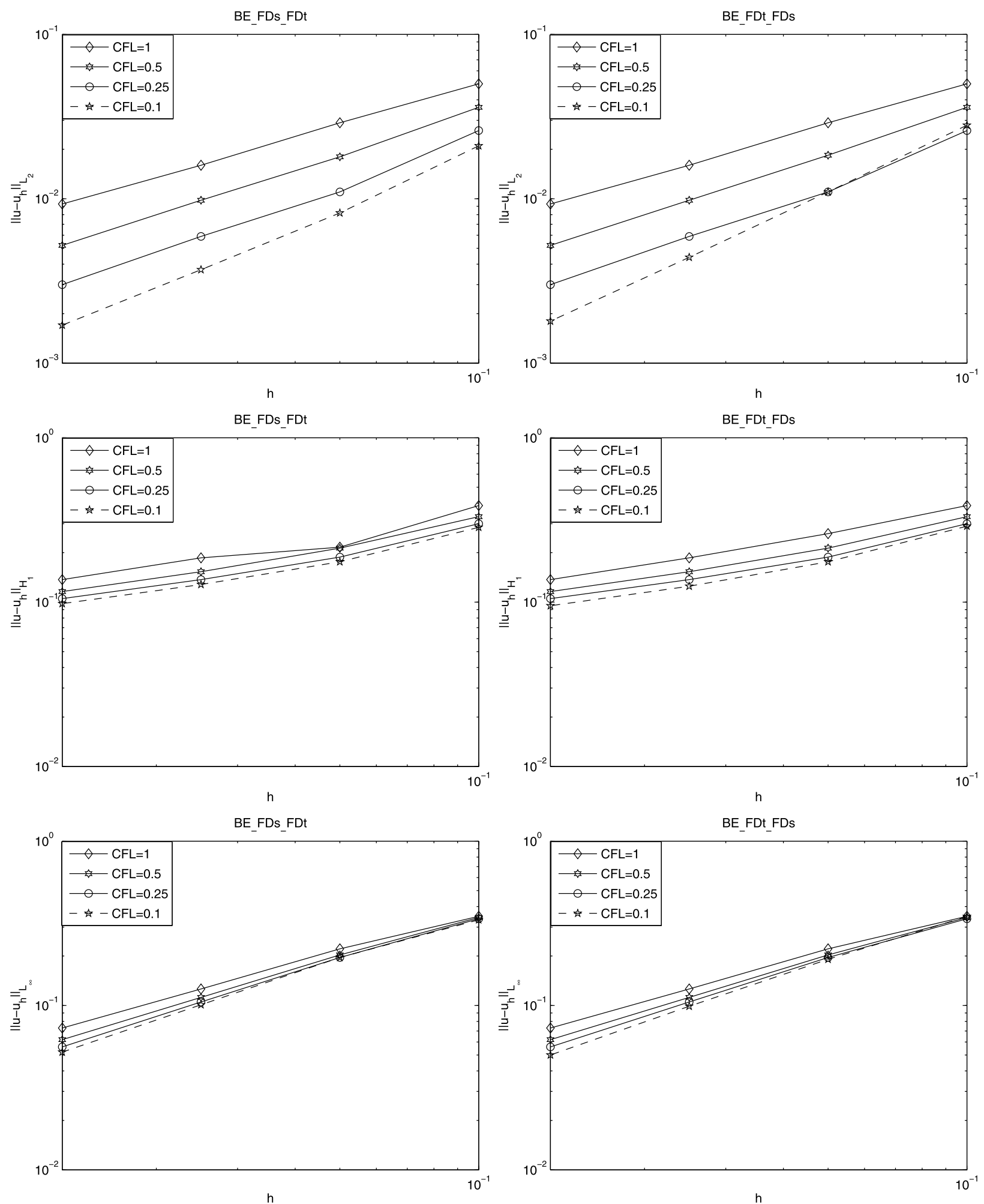

Fig. 5. Test 1 , convergence rates in $L_{2}, H_{1}$ and $L_{\infty}$ norms obtained with Backward Euler time discretization.

show convergence rates in $L_{2}, H_{1}$ and $L_{\infty}$ norms for different CFL numbers at time $t=0.2$. Our first observation is that, $C N_{-} F D t_{-} F D s$ approach performs better at $C F L=0.25$ in $L_{2}$ norm (second graph in Fig. 4) and getting worse as CFL number becomes smaller. This behavior of the method is consistent with characteristic feature of the LCB method (see [1]). Moreover, 


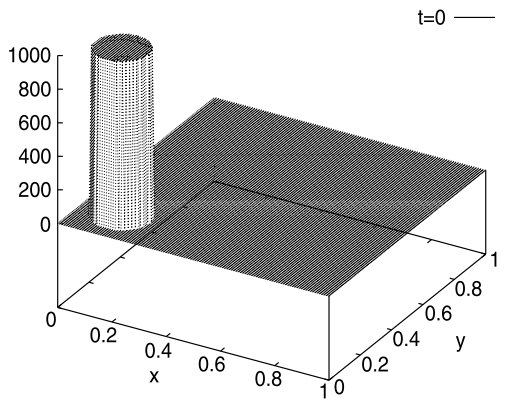

Fig. 6. Test 2, initial condition.
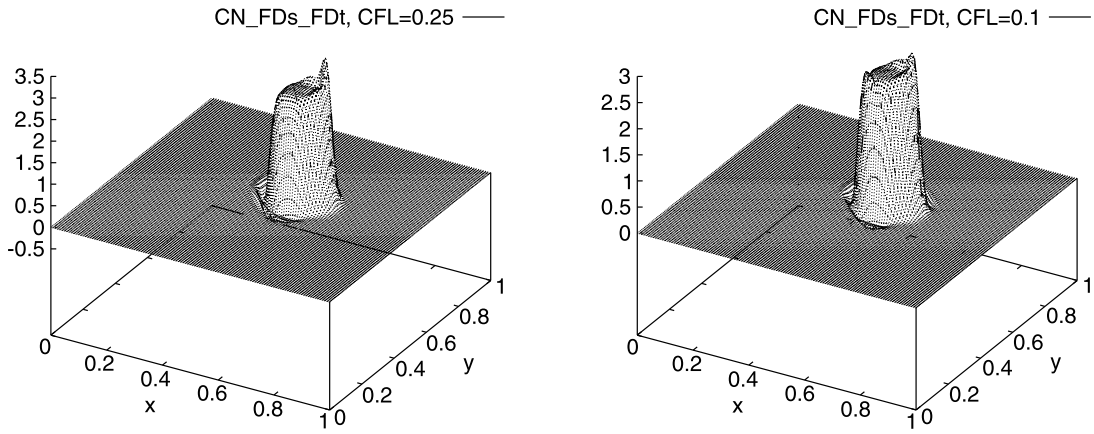

CN_FDt_FDs, $C F L=0.1$
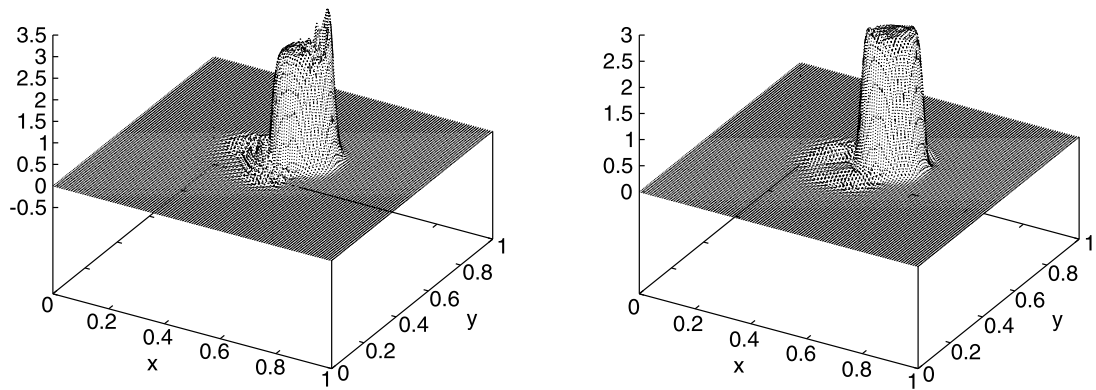

Fig. 7. Test 2, numerical solutions at time $t=0.6$ obtained with Crank-Nicolson scheme combined with FDs_FDt and FDt_FDs approaches.

we can infer from Fig. 4 that there are no significant differences between $C N_{-} F D s_{-} F D t$ and $C N_{-} F D t_{-} F D s$ approaches in $H_{1}$ and $L_{\infty}$ norms. As for the Backward Euler time discretization, we see from Fig. 5 that the same behavior observed for $C N_{-} F D t_{-} F D s$ approach can be seen for $B E_{-} F D t_{-} F D s$ approach in $L_{2}$ norm for large $h$. Again, there exists no considerable difference between $B E_{-} F D s_{-} F D t$ and $B E_{-} F D t_{-} F D s$ approaches in $H_{1}$ and $L_{\infty}$ norms.

\subsection{Test 2}

In this test problem, we consider a moving cylinder which is subject to nonignorable reaction field. The problem data is: $\epsilon=10^{-8},\left(b_{1}, b_{2}\right)=(\cos \pi / 4, \sin \pi / 4), \sigma=10$ and $f=0$. The initial condition is represented in Fig. 6 and defined as follows:

$$
u(0 ; x, y)= \begin{cases}1000 & \text { if } \sqrt{(x-0.15)^{2}+(y-0.15)^{2}} \leq 0.1 \\ 0 & \text { otherwise. }\end{cases}
$$

We employ both Crank-Nicolson and Backward Euler time discretizations combined with FDs_FDt and FDt_FDs approaches. $121 \times 121$ uniform grid points are used for initial discretization. Homogeneous Dirichlet boundary condition is imposed everywhere on the boundary. The difficulty of this problem is that two processes take place at the same time. As time evolves, the cylinder moves in the direction of angle of the wind and height of the cylinder decreases because of the non-ignorable reaction field. It is very difficult to design numerical methods that are able to cope with these two processes, simultaneously. Fig. 7 illustrates numerical solutions obtained with Crank-Nicolson scheme at time $t=0.6$ for $C F L=0.25$ for which 205 time steps are used and for $C F L=0.1$ for which 5128 time steps are used. Fig. 8 shows results obtained 
BE_FDs_FDT, $C F L=0.25$

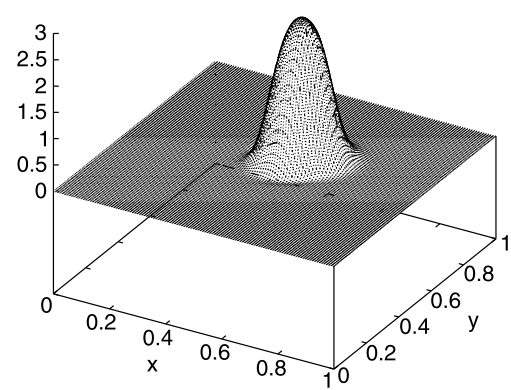

BE_FDt_FDs, $C F L=0.25$

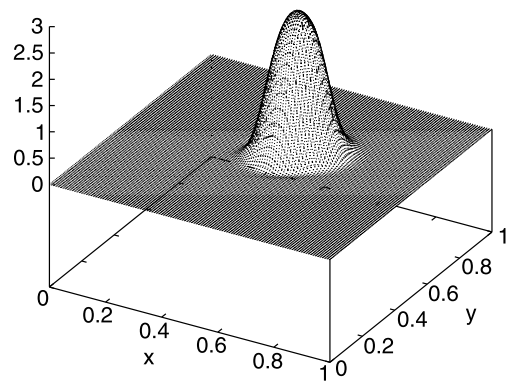

BE_FDs_FDt, $C F L=0.1$

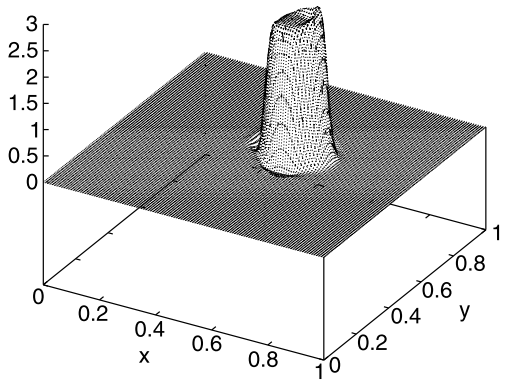

BE_FDt_FDs, CFL $=0.1$

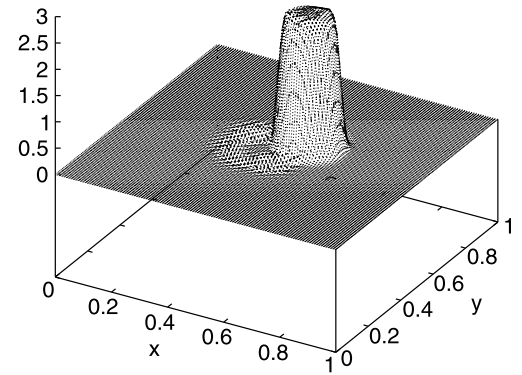

Fig. 8. Test 2, numerical solutions at time $t=0.6$ obtained with Backward Euler combined with FDs_FDt and FDt_FDs approaches.

with Backward Euler scheme for the same parameters. Oscillations near layer regions occurred in the solutions obtained with Crank-Nicolson scheme diminish as CFL number gets smaller. However, FDt_FDs approach leaves some pollutions at the back of the moving body for both time discretizations.

\subsection{Test 3}

We consider an experiment which is studied in [10]. The problem data is: $\epsilon=10^{-6}, b=(1,0), \sigma=0, f=1, u_{0}=0$ and homogeneous Dirichlet boundary condition is imposed everywhere on the boundary. Numerical approximations are obtained with time step $\Delta t=0.001 .21 \times 21$ nodes are used for initial discretization. The solution to this problem has an exponential boundary layer at the outflow boundary $x=1$ and characteristic or parabolic boundary layers at the tangential boundaries $y=0$ and $y=1$. The solutions obtained with $C N_{-} F D s_{-} F D t$ and $C N_{-} F D t_{-} F D s$ approaches are presented in Fig. 9 on structured grid and in Fig. 10 on unstructured grid at time $t=0.6,1.2$. Comparing them with [10, Fig. 3] we see that the results are very similar on both structured and unstructured grids for both $C N_{-} F D s_{-} F D t$ and $C N_{-} F D t_{-} F D s$ approaches.

\subsection{Test 4}

In this test problem we consider non-uniform velocity which was studied in [10]. The problem data is: $\epsilon=10^{-6}, b=$ $(y,-x), \sigma=0, f=1, u_{0}=0$. Time evolves with time step $\Delta t=0.001$. Boundary conditions are: $u=0$ at the boundaries $x=1, y=0, y=1$ and $u=1$ at $x=0.81 \times 81$ nodes are used. In this example, a front is generated at the side $x=0$ and as time evolves, it moves through the domain, thereby rotating around the origin, a curved interior layer is created. Fig. 11 illustrates numerical solutions computed with $C N_{-} F D s_{-} F D t$ and $C N_{-} F D t_{-} F D$ s approaches at times $t=0.6$, 1.2 on uniform grid and Fig. 12 represents the corresponding contour plots. Moreover, Figs. 13, 14 illustrate the results on unstructured grids for the same problem parameters. The numerical solutions in Figs. 11-14 show that the results are consistent with the physical configuration of the problem on both structured and unstructured grids. However, $C N_{-} F D t_{-} F D s$ approach produces small undesired oscillations near layer regions which makes $C N_{-} F D s_{-} F D t$ approach better.

\subsection{Test 5}

This is a benchmark problem which considers a configuration of three geometrical bodies that rotate clockwise. It was studied in $[11,12,16]$. We consider the case $N=129$ and $\Delta t=0.001$ to be comparable with other methods that appeared in literature. The solid body is modeled with a scalar density function that has three shapes; a slotted cylinder, a cone and a sinusoidal hump. The problem data is: $\epsilon=10^{-6}, b=(y-0.5,0.5-x), \sigma=0$ and $f=0$. Homogeneous Dirichlet boundary condition is imposed everywhere on the boundary. The initial condition, consisting of three disjoint bodies, is represented in 

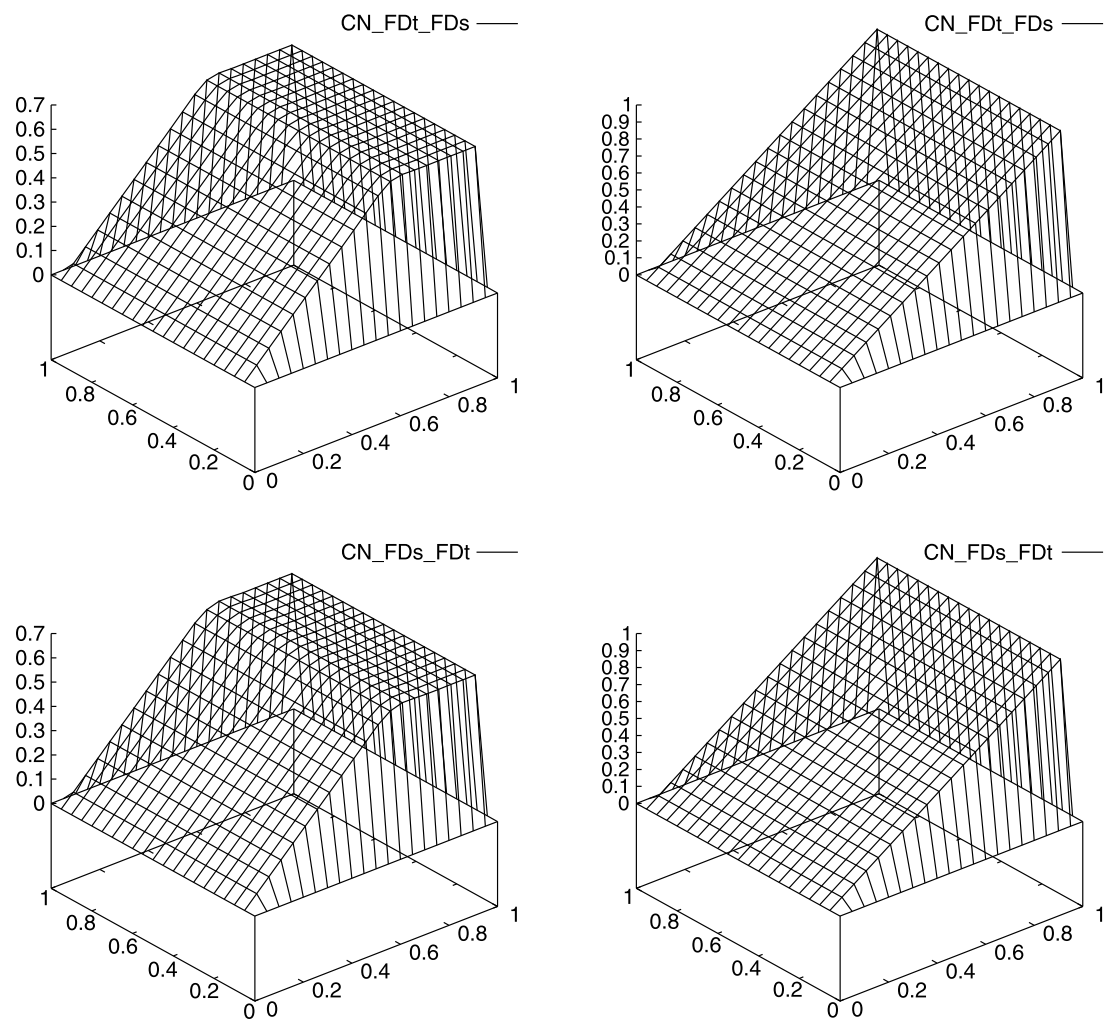

Fig. 9. Test 3, numerical solutions at time $t=0.6$ (left column) and at time $t=1.2$ (right column) obtained with structured grid.
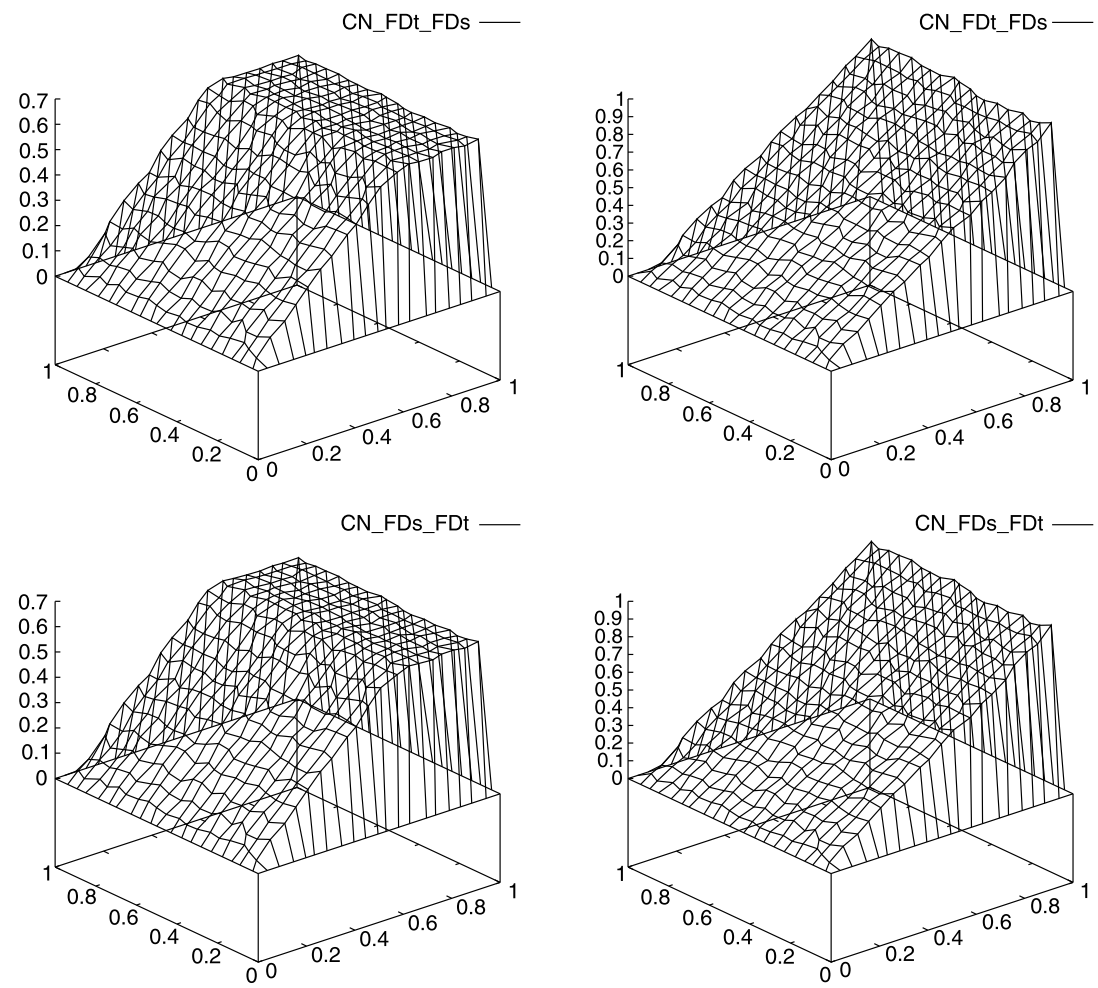

Fig. 10. Test 3 , numerical solutions at time $t=0.6$ (left column) and at time $t=1.2$ (right column) obtained with unstructured grid. 

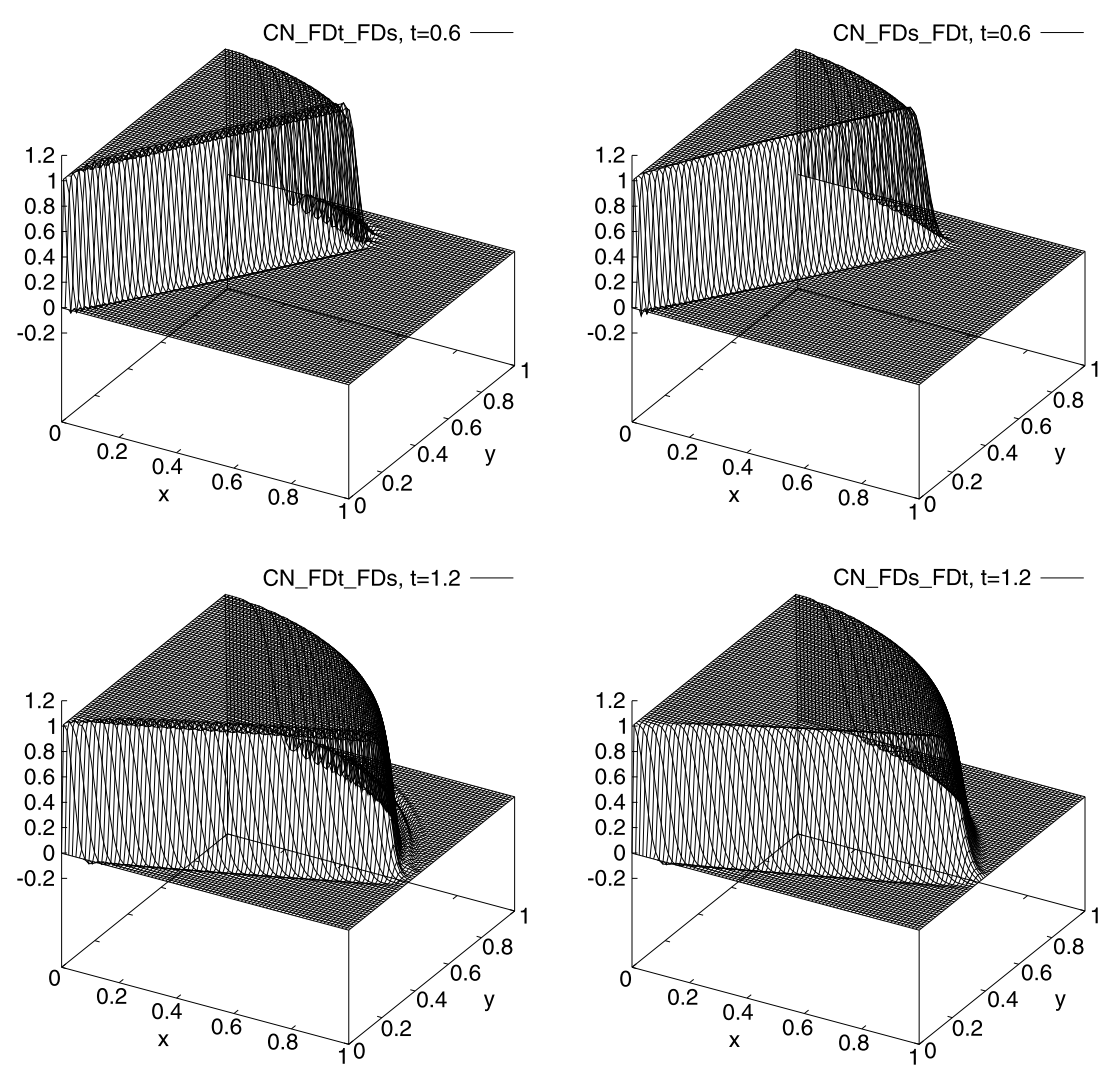

Fig. 11. Test 4 numerical solutions obtained with structured grid.
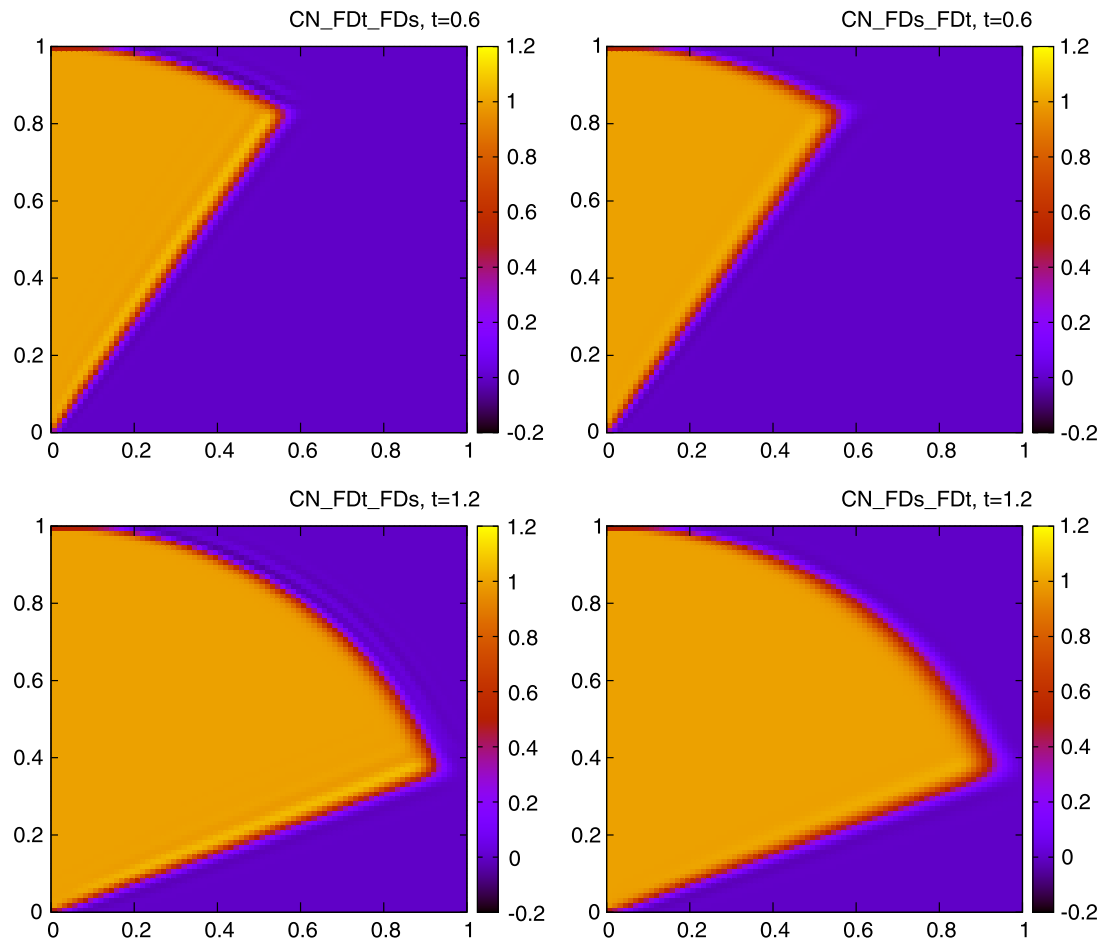

Fig. 12. Test 4, corresponding contour plots of solutions in Fig. 11. 

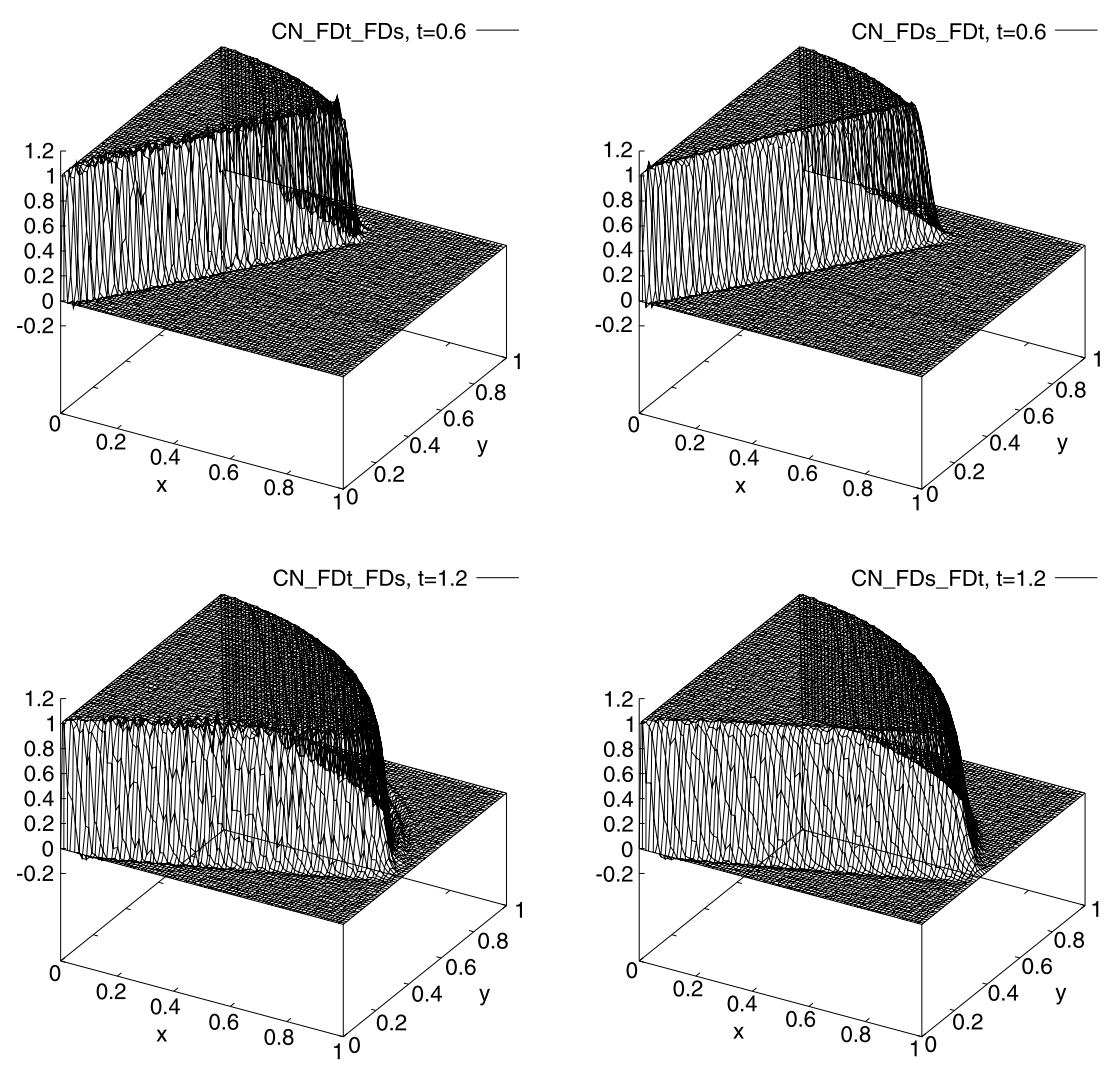

Fig. 13. Test 4 , numerical solutions obtained with unstructured grid.
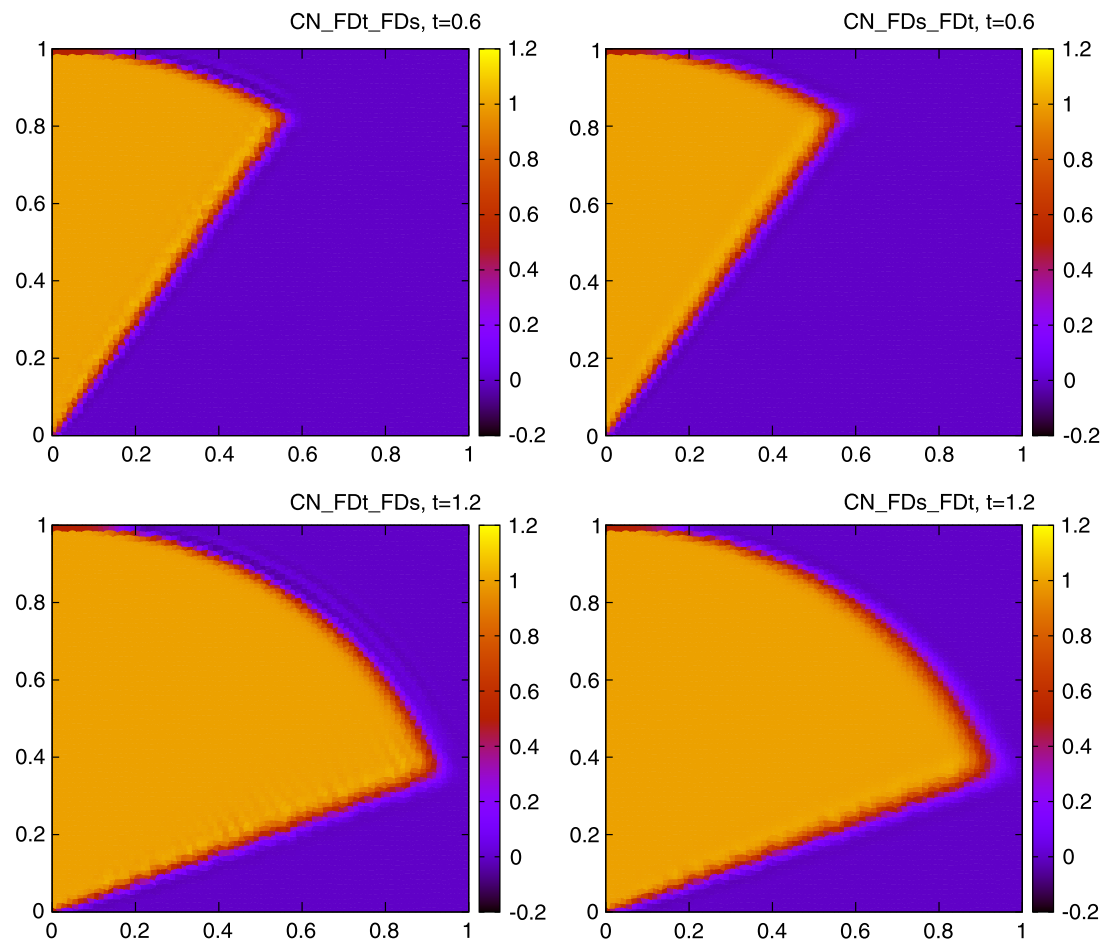

Fig. 14. Test 2, corresponding contour plots of solutions in Fig. 13. 


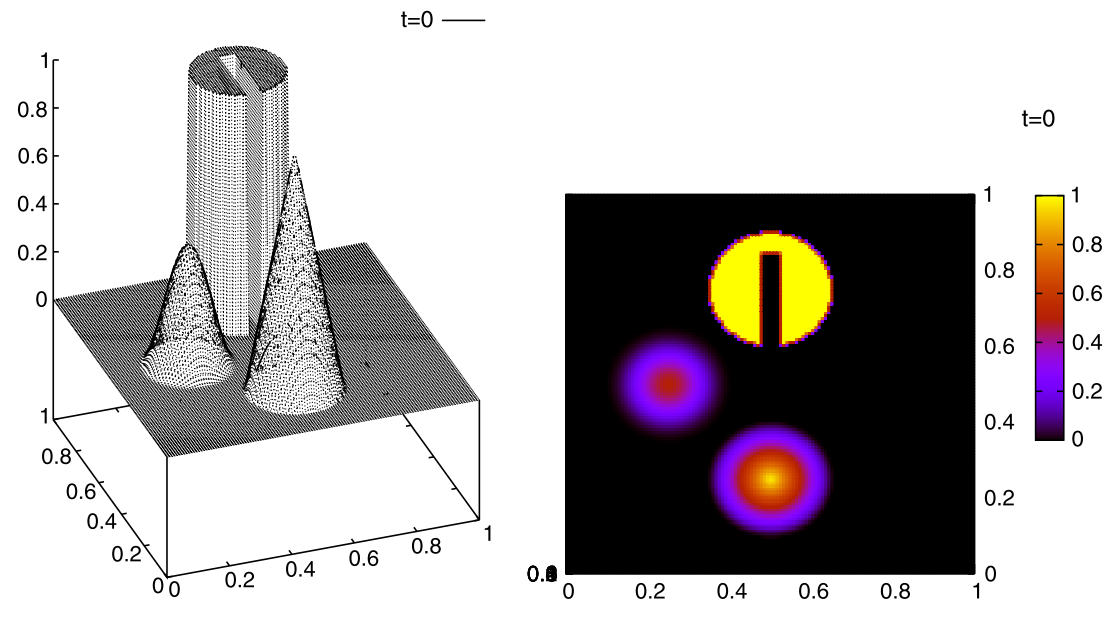

Fig. 15. Test 5, elevation and contour plots of initial condition.

Fig. 15 and it is zero outside the three bodies. More precisely, for a given $\left(x_{0}, y_{0}\right)$, let $r(x, y)=\sqrt{\left(x-x_{0}\right)^{2}+\left(y-y_{0}\right)^{2}} / 0.15$. The center of the slotted cylinder is $(0.5,0.75)$ and it is defined by

$$
u(0 ; x, y)= \begin{cases}1 & \text { if } r(x, y) \leq 1,\left|x-x_{0}\right| \geq 0.0225 \text { or } y \geq 0.85 \\ 0 & \text { otherwise. }\end{cases}
$$

The hump is given by $\left(x_{0}, y_{0}\right)=(0.25,0.5)$ and

$$
u(0 ; x, y)=\frac{1}{4}(1+\cos (\pi \min \{r(x, y), 1\})) .
$$

The cone on lower part of the domain is described by $\left(x_{0}, y_{0}\right)=(0.5,0.25)$ and

$$
u(0 ; x, y)=1-r(x, y) .
$$

Numerical solutions and corresponding contour plots obtained with $C N_{-} F D s_{-} F D t$ and $C N_{-} F D t_{-} F D s$ approaches on uniform grid at time $t=2 \pi$ are presented in Fig. 16. Furthermore, Fig. 17 illustrates the results obtained with the same approaches and the same problem parameters on unstructured grid. We can say from Fig. 16 and Fig. 17 that there exists no significant difference between two approaches on both structured and unstructured grids for this test problem.

\subsection{Test 6}

Our final test problem is devoted for three space dimensional convection-diffusion-reaction equation in unit cube. The problem data is: $\epsilon=10^{-5},\left(b_{1}, b_{2}, b_{3}\right)=(y-0.5,0.5-x, 1), \sigma=0$ and $f=0$. Boundary conditions are: $u=1$ at the boundary $(x, y, z=0)$ and $u=0$ on the rest of the boundary. Initial condition is set to $u_{0}=0$. Time evolves with time step $\Delta t=0.001$. $41 \times 41 \times 41$ nodes are used for initial discretization. We take final time $T=1$. In this example, a front is generated at the bottom of the unit cube and as time advances, it rotates clockwise and moves in the $z$-direction. Fig. 18 represents numerical solutions at the cut planes $z=0.2, z=0.4$ and $z=0.6$ obtained with $C N_{-} F D t \_F D s$ approach on structured grid. Results for $C N_{-} F D s_{-} F D t$ approach are presented in Fig. 19 on structured grid. Moreover, results derived by unstructured grids are illustrated in Fig. 20 for $C N_{-} F D t_{-} F D s$ approach and in Fig. 21 for $C N_{-} F D s_{-} F D t$ approach. We can say that numerical results are consistent with physical configuration of the problem for both approaches both on structured and on unstructured grids. However, small oscillations in the vicinity of internal layers can be observed in the results of $C N_{-} F D t_{-} F D s$ approach.

\section{Conclusion}

In this paper, we have addressed the numerical solution of multidimensional unsteady convection-diffusion-reaction problems with finite difference method on a special grid. The finite difference scheme considered for space discretization is based on [19]. For time discretizations we used Crank-Nicolson and Backward-Euler schemes. Two types of partial differentiation; method of lines and horizontal method of lines were employed. In the numerical tests, we did experiments in $2 D$ and $3 D$ on both structured and unstructured grids and obtained very good results. As we have seen, the method preserves its quality under non-ignorable reaction field. We have observed that $F D t_{-} F D s$ approach (Rothe method) exhibits a similar convergence in $L_{2}$ norm with LCB method for unsteady problem (see [1]). Unlike the results in [1], the best results with the smallest spurious oscillations, have been obtained discretizing first the space variable (method of lines). This is not a contradiction because non-uniform advection which may change superiority of Rothe method with respect to method 
CN_FDt_FDs -

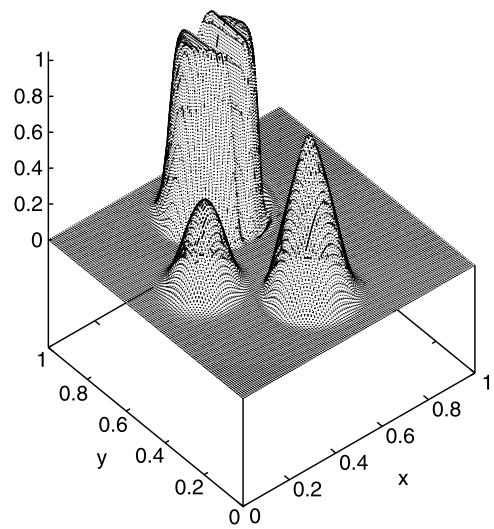

CN_FDt_FDs

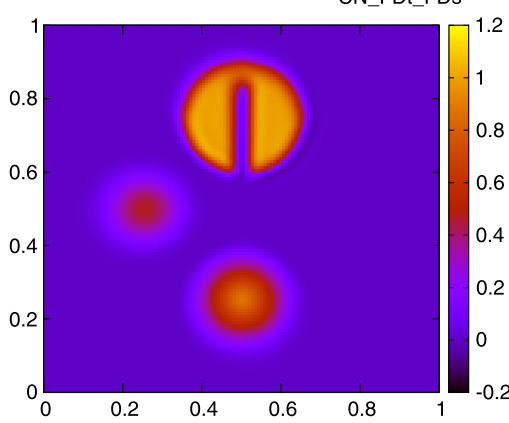

CN_FDs_FDt

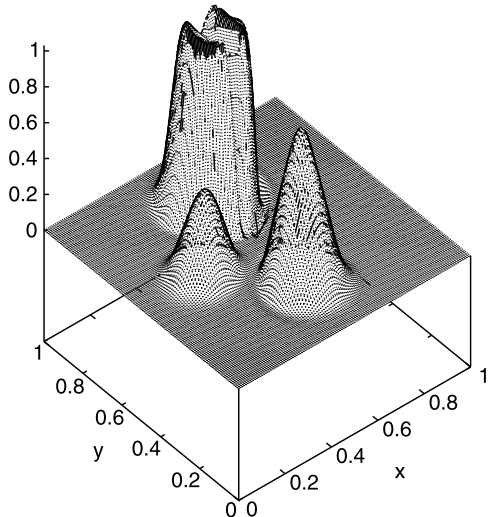

CN_FDs_FDt

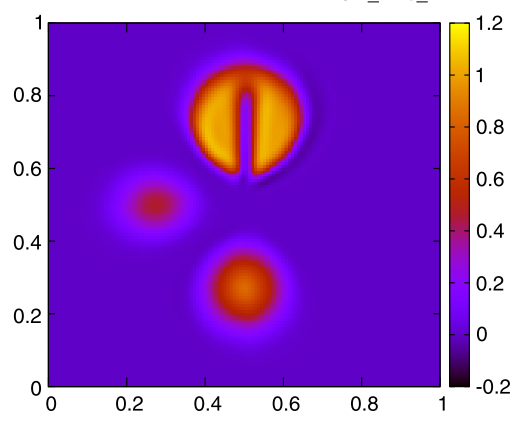

Fig. 16. Test 5, elevation and corresponding contour plots of numerical solutions after a full rotation on structured grid.

CN_FDt_FDs -

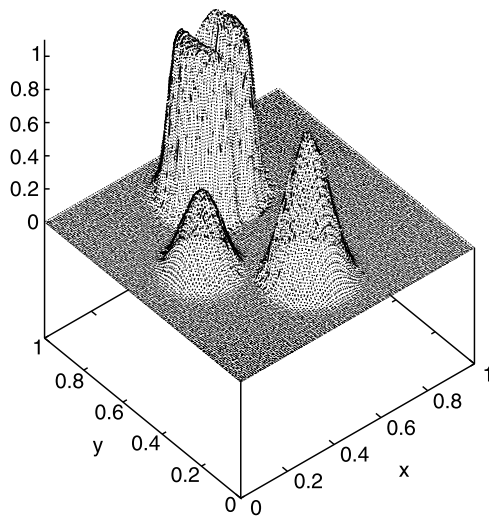

CN_FDt_FDs

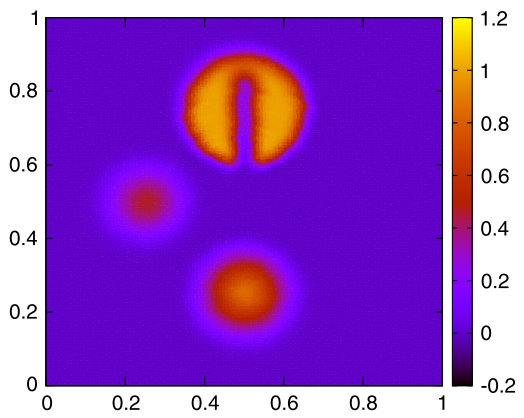

CN FDs FDt -

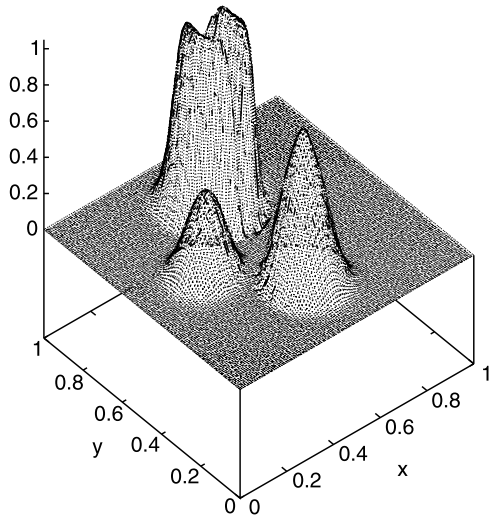

CN_FDs_FDt

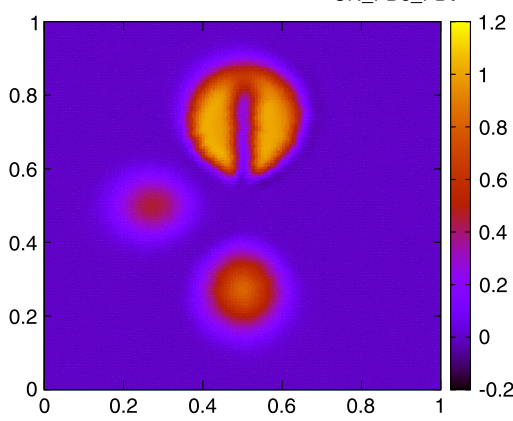

Fig. 17. Test 5, elevation and corresponding contour plots of numerical solutions after a full rotation on unstructured grid. 

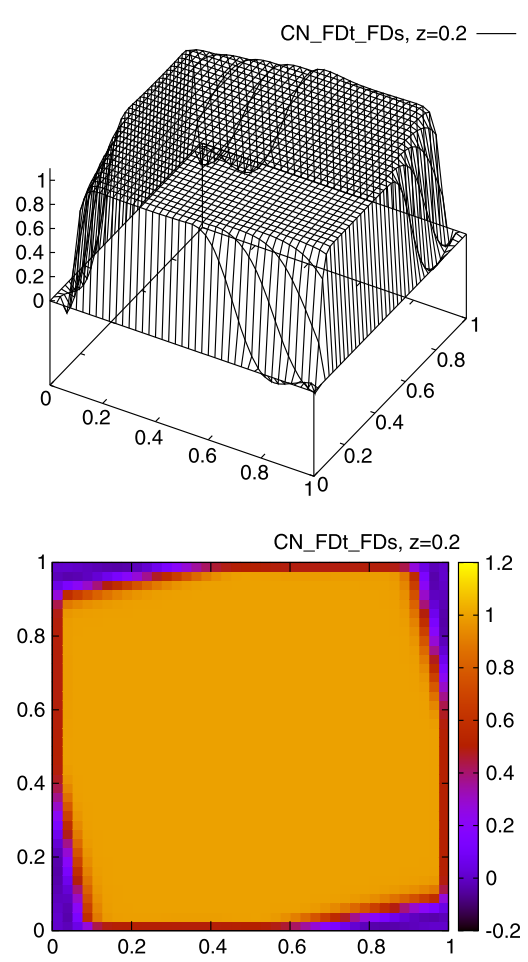

CN_FDt_FDs, $z=0.4$
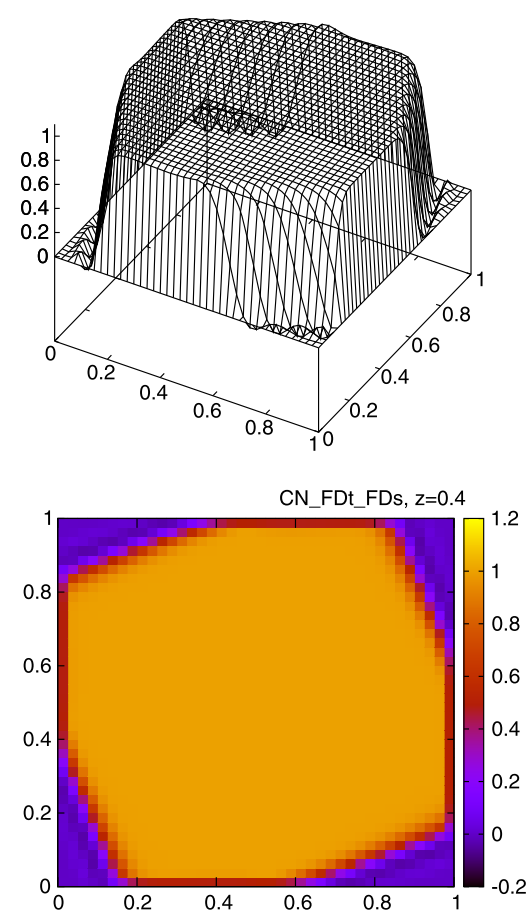

CN_FDt_FDs, $\mathrm{z}=0.6$
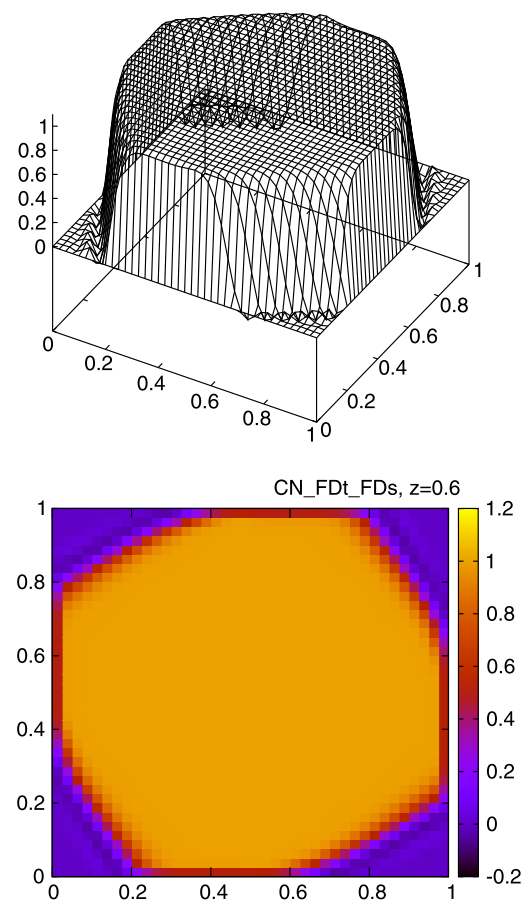

Fig. 18. Test 6, numerical solutions and corresponding contour plots obtained with $C N_{-} F D t_{-} F D$ s approach on uniform grid at the cut planes $z=0.2, z=0.4$ and $z=0.6$.
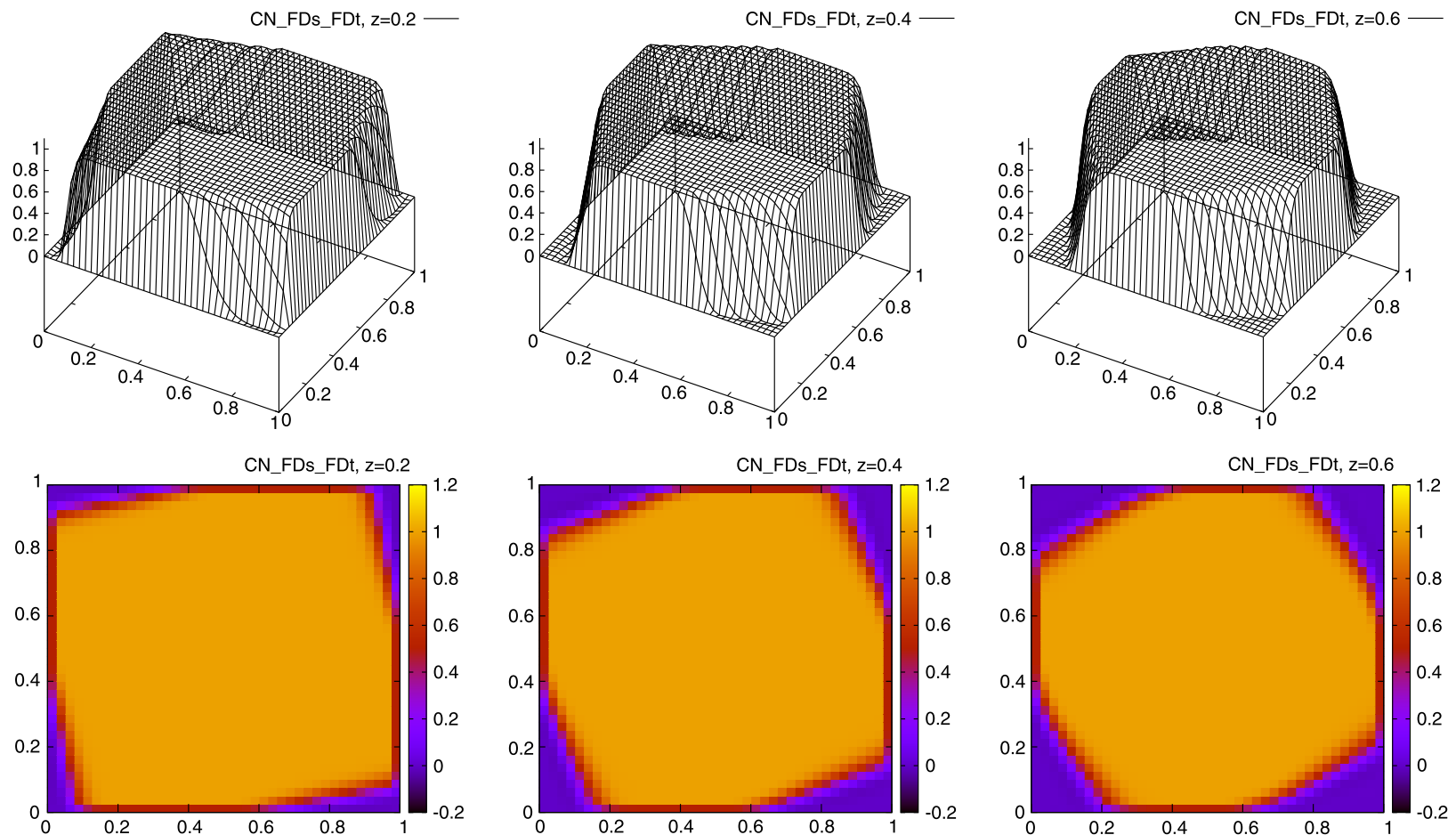

Fig. 19. Test 5, numerical solutions and corresponding contour plots obtained with $C N_{-} F D s_{-} F D t$ approach on uniform grid at the cut planes $z=0.2, z=0.4$ and $z=0.6$. 

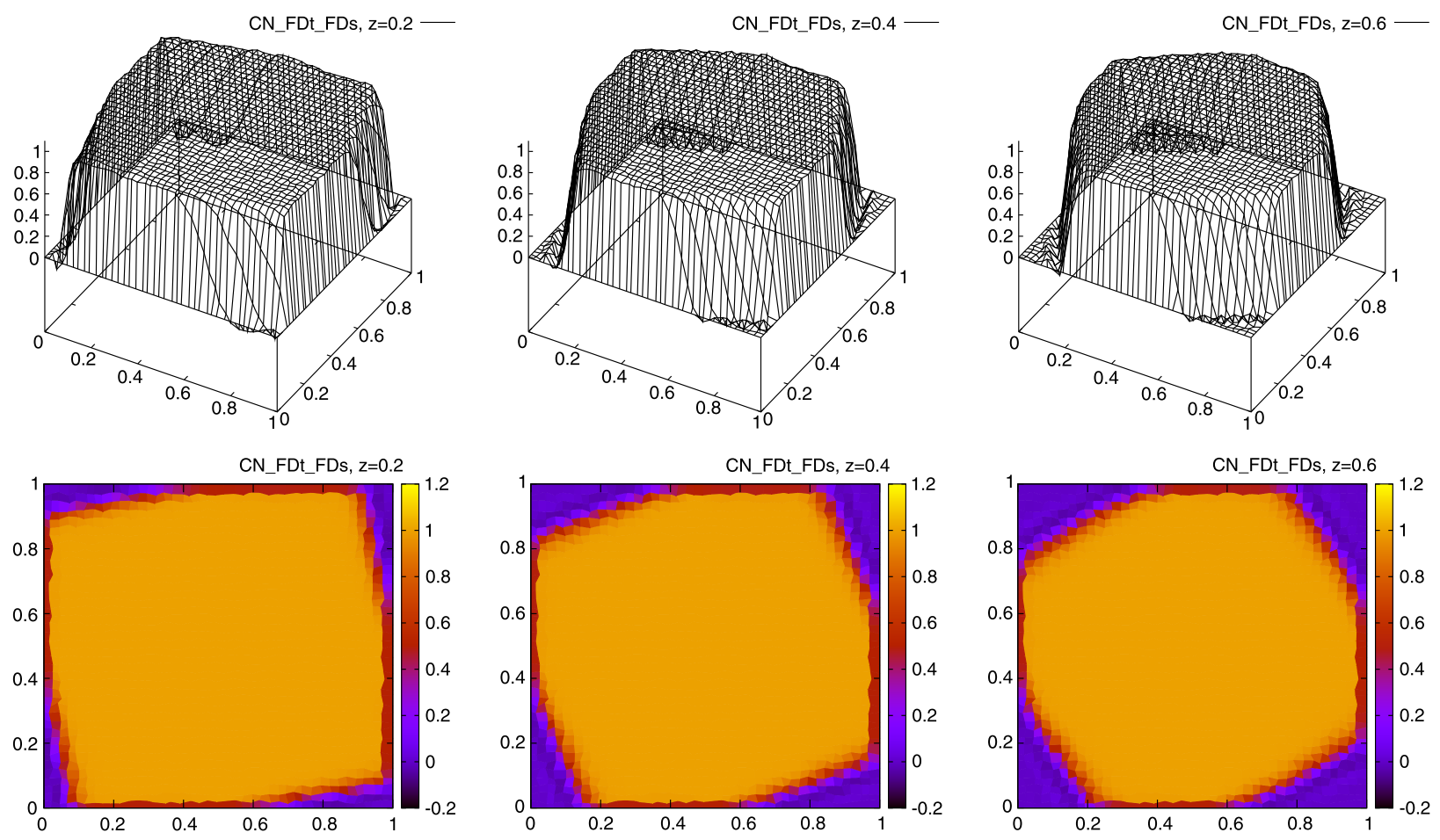

Fig. 20. Test 5 , numerical solutions and corresponding contour plots obtained with $C N_{-} F D t_{-} F D s$ approach on unstructured grid at the cut planes $z=0.2$, $z=0.4$ and $z=0.6$.
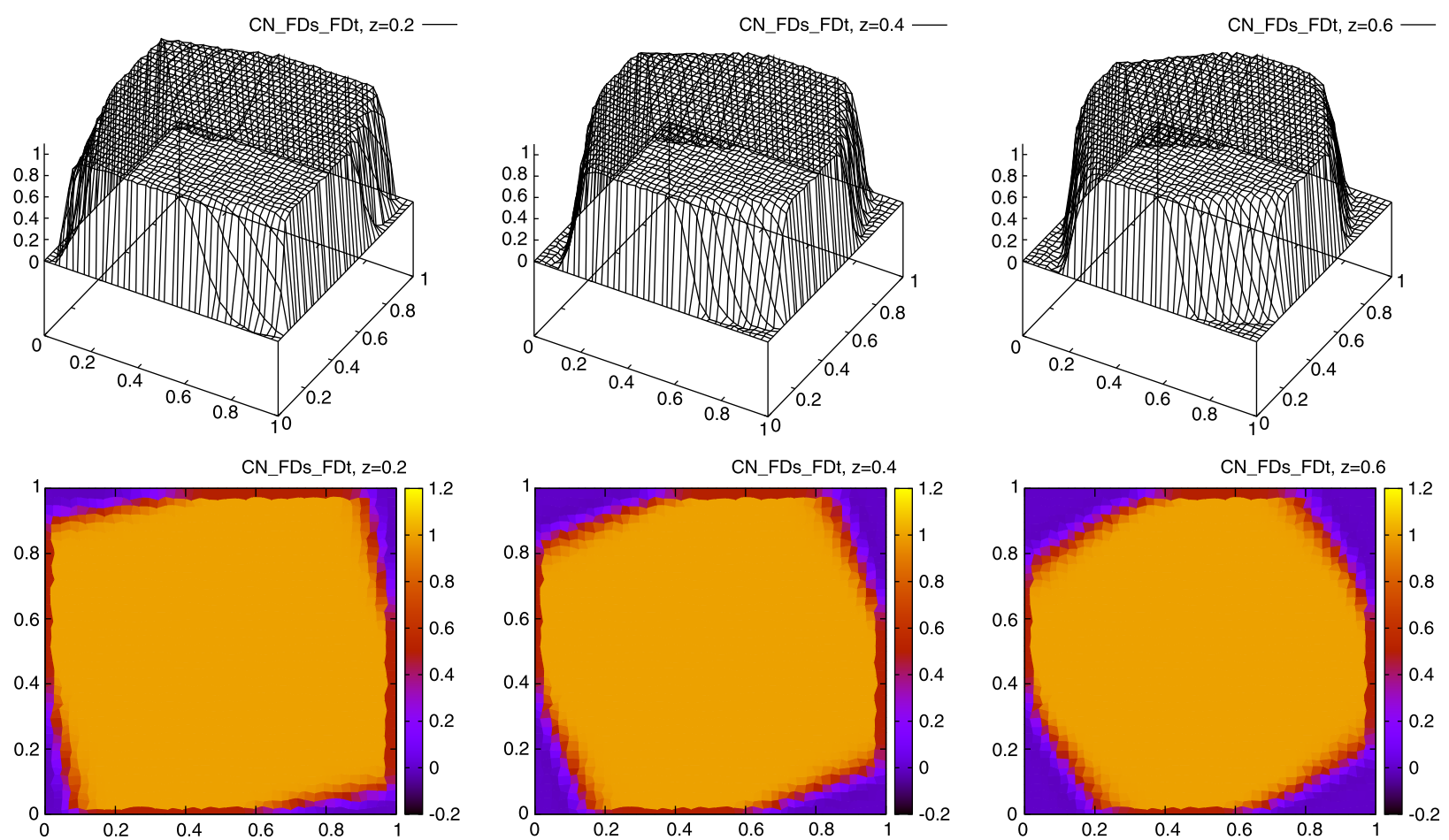

Fig. 21. Test 5 , numerical solutions and corresponding contour plots obtained with $C N_{-} F D s_{-} F D t$ approach on unstructured grid at the cut planes $z=0.2$, $z=0.4$ and $z=0.6$. 
of lines, was not considered in [1]. The results suggest that accuracy of the method proposed in [19] for convection and reaction-dominated regimes in the steady problem allow it to be a good method for unsteady problems.

\section{References}

[1] M.I. Asensio, B. Ayuso, G. Sangalli, Coupling stabilized finite element methods with finite difference time integration for advection-diffusion-reaction problems, Comput. Methods Appl. Mech. Eng. 196 (2007) 3475-3491.

[2] C. Baiocchi, F. Brezzi, L.P. Franca, Virtual bubbles and the GaLS, Comput. Methods Appl. Mech. Eng. 105 (1993) 125-141.

[3] F. Brezzi, G. Hauke, L.D. Marini, G. Sangalli, Link-cutting bubbles for the stabilization of convection-diffusion-reaction problems, Math. Models Methods Appl. Sci. 13 (2003) 445-461.

[4] F. Brezzi, L.D. Marini, A. Russo, Applications of pseudo residual-free bubbles to the stabilization of convection-diffusion problems, Comput. Methods Appl. Mech. Eng. 166 (1998) 51-63.

[5] F. Brezzi, L.D. Marini, A. Russo, On the choice of a stabilizing subgrid for convection-diffusion problems, Comput. Methods Appl. Mech. Eng. 194 (2004) $127-148$.

[6] A.N. Brooks, T.J.R. Hughes, Streamline upwind/Petrov-Galerkin formulations for convection dominated flows with particular emphasis on the incompressible Navier-Stokes equations, Comput. Methods Appl. Mech. Eng. 32 (1982) 199-259.

[7] L.P. Franca, A.I. Neslitürk, M. Stynes, On the stability of residual-free bubbles for convection-diffusion problems and their approximation by a two-level finite element method, Comput. Methods Appl. Mech. Eng. 166 (1998) 35-49.

[8] A. Şendur, A.I. Neslitürk, Applications of the pseudo residual-free bubbles to the stabilization of convection-diffusion-reaction problems, Calcolo 49 (2011) 1-19.

[9] J. de Frutos, B. García-Archilla, V. John, J. Novo, An adaptive finite element method for evolutionary convection dominated problems, Comput. Methods Appl. Mech. Eng. 200 (2011) 3601-3612.

[10] J. de Frutos, B. García-Archilla, V. John, J. Novo, An adaptive SUPG method for evolutionary convection-diffusion equations, Comput. Methods Appl. Mech. Eng. 273 (2014) 219-237.

[11] V. John, E. Schmeyer, Finite element methods for time-dependent convection-diffusion-reaction equations with small diffusion, Comput. Methods Appl. Mech. Eng. 198 (3-4) (2008) 475-494.

[12] N. Ahmed, G. Matthies, L. Tobiska, H. Xie, Discontinuous Galerkin time stepping with local projection stabilization for transient convection-diffusionreaction problems, Comput. Methods Appl. Mech. Eng. 200 (2011) 1747-1756.

[13] A. Kaya, A finite difference scheme for multidimensional convection-diffusion-reaction equations, Comput. Methods Appl. Mech. Eng. 278 (2014) 347-360.

[14] R. Codina, Comparison of some finite element methods for solving the diffusion-convection-reaction equation, Comput. Methods Appl. Mech. Eng. 156 (1998) 185-200

[15] G. Lube, D. Weiss, Stabilized finite element methods for singularly perturbed parabolic problems, Appl. Numer. Math. 17 (1995) $431-459$.

[16] V. John, J. Novo, On (essentially) non-oscillatory discretizations of evolutionary convection-diffusion equations, J. Comput. Phys. 231 (2012) 1570-1586.

[17] A. Harten, B. Enquist, S. Osher, S. Chakravarthy, Uniformly high order essentially non-oscillatory schemes III, J. Comput. Phys. 71 (1987) $231-303$.

[18] X.-D. Liu, S. Osher, T. Chan, Weighted essentially non-oscillatory schemes, J. Comput. Phys. 115 (1994) $200-212$.

[19] A. Kaya, A. Sendur, Finite difference approximations of multidimensional convection-diffusion-reaction problems with small diffusion on a special grid, 2014, JCOMP-D-14-01145. 